\title{
Unsteady Aerodynamic Model of Flexible Flapping Wing
}

\author{
Si Chen ${ }^{1,2}$, Hao Li ${ }^{2}$, Shijun Guo ${ }^{2 *}$, Mingbo Tong ${ }^{1}$, Bing $\mathbf{J i}^{3}$ \\ ${ }^{1}$ College of Aerospace Engineering, Nanjing University of Aeronautics and \\ Astronautics, Nanjing, P.R. China \\ ${ }^{2}$ School of Aerospace, Transport and Manufacturing, Cranfield University, Cranfield, \\ UK \\ ${ }^{3}$ School of Control Science and Eng, Shandong University, Jinan, P.R. China \\ E-mail: s.guo@cranfield.ac.uk
}

\begin{abstract}
Bio-inspired flapping wing has potential application to micro air vehicles (MAV). Due to the nature of lightweight and flexibility of micro flapping wing structures, elastic deformation as a result of aeroelastic coupling is inevitable in flapping motion. This effect can be significant and beneficial to the aerodynamic performance as revealed in the present investigation for a flexible flapping wing of variable camber versus a rigid one. Firstly a two dimensional (2D) unsteady aerodynamic model (UAM) based on potential flow theory has been extended from previous study. Both leading and trailing edge discrete vortices are included in the model with unsteady Kutta condition satisfied to fully characterize the unsteady flow around a flapping wing. A wall function is created to modify the induced velocity of the vortices in the UAM to solve the vortices penetration problem. The modified UAM is then validated by comparing with CFD results of a typical insect-like flapping motion from previous research. Secondly the UAM is further extended for a flexible flapping wing of camber variation. Comparing with a rigid wing in a prescribed plunging and pitching motion, the results show lift increase with positive camber in upstroke by mitigating negative lift. The results also agree well with CFD simulation. Thirdly the 2D UAM is extended to calculate the aerodynamic forces of a 3D wing with camber variation, and validated by CFD results. Finally the model is applied to aerodynamic analysis of a 3D flexible flapping wing with aeroelastic coupling effect. Significant increase of lift coefficient can be achieved for a flexible flapping wing of positive camber and twist in upstroke produced by the structure elastic deformation.
\end{abstract}

Keywords: Unsteady aerodynamic model, Flapping wing, Variable camber, Aeroelastic coupling.

\section{Introduction}

Theoretical study on flapping wing was started since 1920s when W. Birnbaum investigated the reaction of an aerofoil in small amplitude oscillation[1]. Over the past several decades, with the great interest in unmanned micro air vehicles (MAV) development, more attention has been paid to smallscale flapping wing models[2]. Due to the small scale and light weight of MAVs (wingspan $\leq 15 \mathrm{~cm}$, 
weight $\leq 20 \mathrm{~g}$ )[3], a typical flapping wing model consists of thin aerofoil and flexible wing structure, and the fluid flow associated with the wing in the low Reynolds number $\left(\operatorname{Re} \sim 10^{3}\right)$ region is often governed by the formation and shedding of vortices from the leading and trailing edges. In recent years, the flow mechanism associated with the flapping wings has been investigated extensively. Dickinson and Götz [4] measured the forces on an impulsively started airfoil at $\operatorname{Re} \sim 100$, and showed that lift was increased by $80 \%$ due to the presence of a leading edge vortex (LEV) in the first 2 chord length of travel. Ellington et al [5] proposed that the insect wings make use of a spanwise flow which serves to transport vorticity of the LEV core along the wingspan, thus stabilizes the LEV and produce consistently high lift. Guo et al [6] conducted a theoretical study, numerical modelling and experiment of a micro flapping wing rotor (FWR) to demonstrate the FWR advantages in simple and light weight configuration and high performance. Wang et al [7] used numerical simulation to model the forces and flow of a flapping $2 \mathrm{D}$ aerofoil and showed that significant lift can be obtained for the wing within 2-4 chord lengths of travel by making use of the dynamic stall of the LEV. Li et al [8] have extended the study of aerodynamic lift and efficiency of insect flapping wing in comparison with rotary and a novel flapping wing rotor.

Due to the complex flow structure of the flapping wing, numerical modelling of the forces and flow dynamics needs to take into account the vortices shedding at the leading and trailing edges. In previous studies [9-11], computational fluid dynamics (CFD) method have been applied to investigate the unsteady forces and flow. However, given the complex flow structure associated with flapping wing, the CFD method often requires relatively large computational time. On the other hand, potential flow based analytic approaches could offer simple and efficient solutions to the flow phenomena. Katz and Allen[12] proposed a vortex panel method to simulate an unsteady 2D aerofoil. In their model, a separated shear layer is set up at a distance behind the leading edge and the separation point is assumed to be fixed at $5 \%$ chord from the leading edge. By using this method, they obtained the results of periodic wake shedding for an inclined aerofoil. Hammer et al[13] extended Katz and Plotkin's method by assuming that the strength of leading edge vortex shedding is proportional to the 
strength of the bound vortex nearest to the leading edge. They adapted the method by Uhlman [14] to calculate the fluid forces, which showed good agreement with experimental and CFD results for a range of wing pitch-hold motion cases. Taha et al[15] developed an aerodynamic model in state-space and validated the model by comparing with a N-S based direct numerical simulation (DNS) results of insect hovering. The study also included an assessment of the leading edge vortices dominance on aerodynamic forces of flapping wing and derived a reduced-order model from the full model. Yan et al[16] developed a semi-analytical unsteady potential flow model and validated their model against the experimental and computational results. This kind of semi-analytical unsteady potential flow model is then employed to find the lift frequency response at different angle of attack. Lam [17] and Hewett [18] obtained an analytic solution of vortices distribution on a steady aerofoil and superimpose the unsteady discrete vortices terms to find the final results on complex plane. Their model was validated against several steady cases. In order to take into account the shedding of leading edge vortex, Ansari et al[19] proposed another set of equations to extend this model by taking leading edge vortices shedding into consideration where Kutta-Joukowski condition at trailing edge and stagnation condition at leading edge are enforced to find the strength of leading edge vortices and trailing edge vortices. Both the force data and the flow field representation obtained from the model showed good agreement with the Robo-fly data from experiment done by Dickinson et al[20].

For small-scale flapping wings, relatively large elastic deformation to the wing scale is inevitable due to the nature of light weight wing structure and high frequency motion. As the deformation interacts with the aerodynamic force, aeroelastic coupling plays significant role in the aerodynamic performance of flapping wings. In previous studies, the effect of flexibility of flapping wings has also been investigated. Hu et al [21] carried out an experimental investigation on aerodynamic performance of a flexible flapping wing and noted that flapping motion can significantly benefit the aerodynamic forces when the advance ratio is smaller than 1 . They also found that the flexible membrane wings have better aerodynamic performance for soaring flight and the flexible wing was found to have better thrust generation performance for while rigid wing can generate higher lift for flapping wing in 
general. Du and Sun [22] solved Navier-Stokes equations on a dynamically deformed grid. The study proposed that the effect of combined camber and twist deformation is similar to that of camber deformation. With a deformation of $6 \%$ camber and $20^{\circ}$ twist, lift is increased by $10 \%-20 \%$ and liftto-drag ratio by around $10 \%$ compared with the case of a rigid flat-plate wing. Guan and $\mathrm{Yu}[23,24]$ used in-house panel method and found that all of twisting, cambering and bending have positive influence on the lift by comparing cases in a parametric study. They also found that lift and thrust are generated mainly during downstroke and negligible during upstroke by the integrated morphing model-wing. Ghommem et al $[25,26]$ used the unsteady vortex lattice method (UVLM) to investigate the wing shape effect on flapping wing propulsion. By parameterizing the twisting and bending modes of the wing in trigonometric and spline-based polynomials, the results show that a proper morphing of the wing can greatly increase the thrust production. In addition, an optimal wing shape for maximum propulsive efficiency was also obtained using a gradient-based optimization.

In previous study, a nonlinear unsteady aerodynamic model (UAM) was developed for a rigid flapping wing [19]. Since the UAM is based on potential flow theory, we appreciate that the viscosity effect is not included hence the aerodynamic results will not affected by Reynolds number. In the model, both leading and trailing edge vortices have bene included. A numerical approach was attempted to reduce the amount of free vortices penetration through the airfoil surface by increasing the airfoil boundary discretization. This problem becomes more severe for a flapping wing in hovering where little freestream flow exists to drive the shed vortices away. Inevitably the vortices shed from the wing in a down-stroke motion will impact on the following up-stroke wing, and vice versa. This was experienced in the present study when adapting the model developed in previous study by Lam [17], Hewett [18] and Ansari et al[19]. Nevertheless the vortices penetration problem has not been solved in the previous study. In the present study, a collision-avoidance algorithm is developed by enforcing a zero-through-flow boundary condition in the model. The results show no vortices penetration occurring in the model and a much better agreement with CFD results obtained by Wang [27] in terms of lift and drag coefficients. This is the first contribution made in the present study to 
extend the UAM for a flapping wing of a prescribed aerofoil shape and presented in Section 2. In order to simulate a flexible flapping wing, the model is further extended to take into account instantaneous shape variation and induced velocity of a flexible wing boundary during flapping motion. This extended model is capable of evaluating aeroelastic coupling effect and performing optimization of a flexible flapping wing. This is the second contribution made in the present study and presented in Section 3. The model is validated by comparing the lift coefficients with CFD analysis results for a 2D wing of rigid aerofoil shape and one of variable camber. The results show that the average lift coefficient in a flapping cycle can be significantly increased by modulating the flexible wing camber in upstroke. The study is then extended to a 3D wing model with camber variation and compared with CFD results. The results from both rigid and flexible wings using the model are compared with CFD results[7,27-29]. Finally, aeroelastic coupling effect is counted in the analysis with the aerodynamic model interacting with structural model (Abaqus solver) for the 3D wing model. The results show significant increase of lift coefficient for a flapping wing with positive camber and twist in upstroke versus a rigid wing. A conclusion of the study is drawn in Section 4.

\section{Theoretical Basis and Methods}

\subsection{Joukowski transform for cambered airfoil}

As shown in Figure 1, for a cambered airfoil, the classical Joukowski transformation can be applied to uniquely map a circle of radius $R$ in the z-plane into an airfoil in the $\zeta$-plane. According to Hewett[18], the transformation can be given by the equation as follows:

$$
\zeta=\mathrm{z}+\frac{(1-\varepsilon) R^{2}}{z}+\frac{R^{3} \varepsilon}{2 z^{2}}, \quad \varepsilon=\frac{(\tau-i \sigma)}{R}
$$

where $\zeta$ and $\mathrm{z}$ are the complex coordinates in the $\zeta$-plane and the $\mathrm{z}$-plane respectively; $\tau$ and $\sigma$ are non-dimensional factors governing the thickness and the camber of the airfoil[18]. Equation (1) can also be written in $\zeta$-plane,

$$
\xi=2 R \cos \theta+\sigma\left(\sin \theta-\frac{1}{2} \sin 2 \theta\right)-\tau\left(\cos \theta-\frac{1}{2} \cos 2 \theta\right)
$$




$$
\eta=\tau\left(\sin \theta-\frac{1}{2} \sin 2 \theta\right)+\sigma\left(\cos \theta-\frac{1}{2} \cos 2 \theta\right)
$$

where $\theta$ is the angular displacement about the origin of the z-plane; $\xi$ is the abscissa of a point on the $\zeta$-plane while $\eta$ is the ordinate of a point on the z-plane.

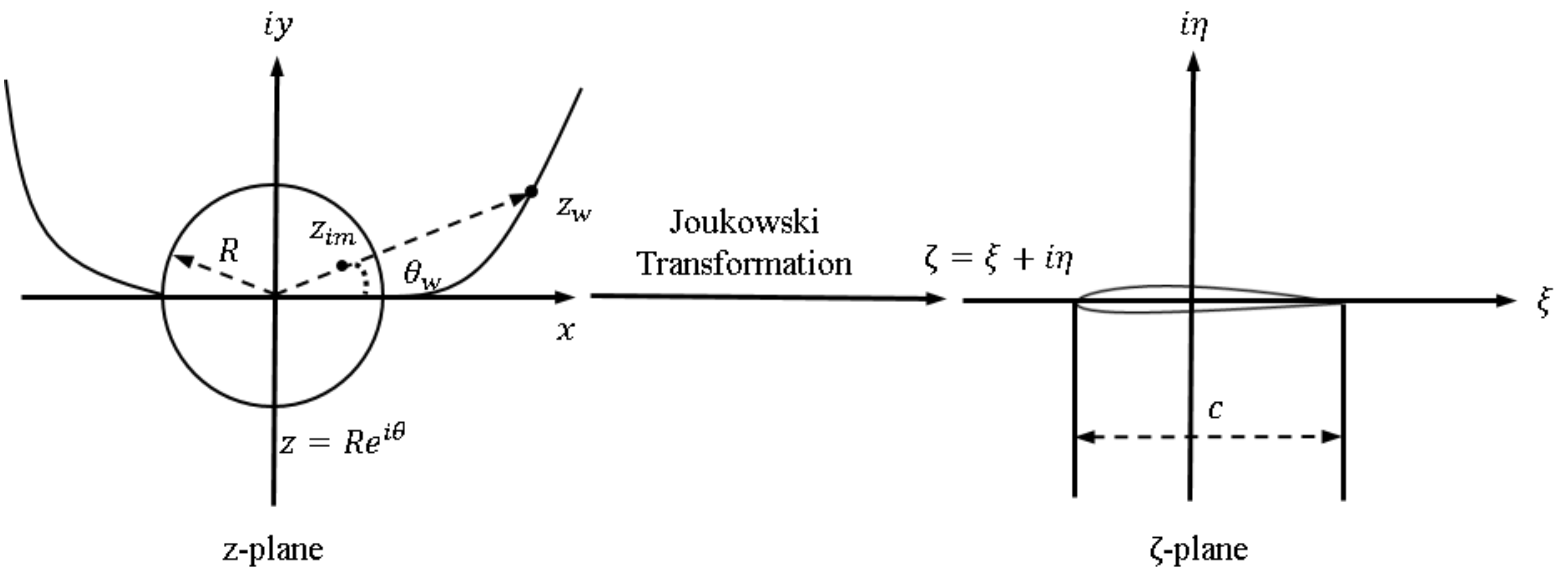

Figure 1. Joukowski Transformation of 2D airfoil

\subsection{Unsteady boundary condition for variable wing camber}

A 2D rigid body airfoil motion in the 2D plane can be represented by heaving $(h, \dot{h})$, sweeping $(l, \dot{l})$ and pitching $(\alpha, \dot{\alpha})$ motion. For the airfoil with variable camber, an additional camber variable $(\sigma, \dot{\sigma})$ is used to describe the motion of the airfoil as shown in Figure 2. The zero-through-flow boundary condition enforced on the airfoil boundary can therefore be written as:

$$
\begin{gathered}
u=\dot{l} \cos \alpha-\dot{h} \sin \alpha+\eta \dot{\alpha}+\dot{\sigma}(\sin \theta-0.5 \sin 2 \theta) \\
v=\dot{l} \sin \alpha+\dot{h} \cos \alpha-\xi \dot{\alpha}+\dot{\sigma}(\cos \theta-0.5 \cos 2 \theta)
\end{gathered}
$$

where $u$ and $v$ are real part and imaginary part of the complex velocity $(q=u-i v)$ at a point on the airfoil and $\theta$ is the angular displacement about the origin of the z-plane. $\sigma$ and $\dot{\sigma}$ represent a nondimensional camber and its variation rate of the airfoil. 


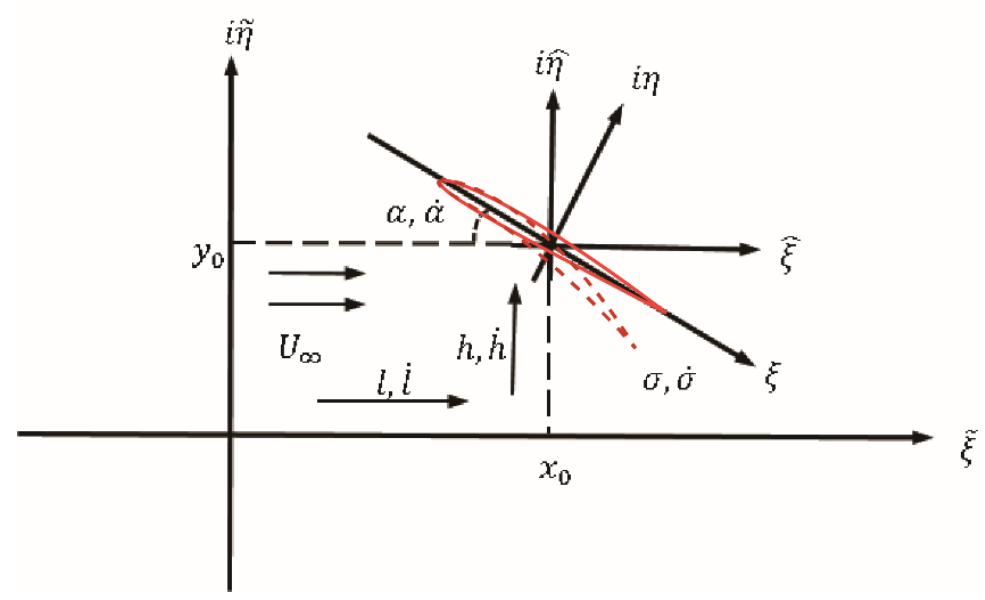

Figure 2. 2D flapping airfoil in a complex coordinate system

\subsection{Potential flow solution using discrete vortices}

In order to model the unsteady $2 \mathrm{D}$ flow over the airfoil, discrete vortices are modelled in both the leading edge and trailing edge. At each time step, the velocity potential is required to satisfy the unsteady boundary condition which incorporates both the rigid body motion and the induced velocity due to wing camber variation. In this model, the flow potential is divided into two terms [19]: the quasi-steady term and the unsteady term. The quasi-steady term is relevant to the freestream and airfoil motion, while the unsteady term is related to the roll-up of the leading and trailing edge vortices. The total potential of the flow at each time step is then obtained by summing these individual terms.

\subsubsection{Quasi-steady potential solution}

Consider an airfoil moving horizontally and vertically with velocity $\dot{l}$ and $\dot{h}$, and the freestream has horizontal velocity $U_{\infty}$, as shown in Figure 2 . The velocity potential for the freestream around a circle of radius $R$ in the z-plane is given by [19]:

$$
\Phi_{f}(z)=U_{\infty}\left(z e^{-i \alpha}+\frac{R^{2} e^{i \alpha}}{z}\right)+\frac{\Gamma_{k}}{2 \pi i} \ln (z)
$$

The circumferential velcotiy caused by freestream can be obtained by differentiating the potential function with respect to $z$ : 


$$
v_{\theta f}(\theta)=-2 U_{\infty} \sin (\theta-\alpha)+\frac{\Gamma_{k}}{2 \pi R}
$$

By reinforcing the Kutta-Joukowski condition $\left(v_{\theta f}(\theta)=0\right.$ when $\left.\theta=0\right)$ at trailing edge, the value of circulation due to freestream can be derived and represented as:

$$
\Gamma_{k}=-4 \pi R U_{\infty} \sin \alpha
$$

Based on the zero-flow-through boundary conditions stated in equation (4) and (5), the radial velocity on the circle in the z-plane due to airfoil motion is obtained as:

$$
\begin{aligned}
& v_{r} \\
& =\frac{1}{R}\left(A_{1} \sin \theta+A_{2} \sin 2 \theta+A_{3} \cos \theta+A_{4} \cos 2 \theta+A_{5} \sin ^{2} \theta+A_{6} \cos ^{2} \theta+A_{7} \sin \theta \cos \theta\right. \\
& +A_{8} \sin \theta \sin 2 \theta+A_{9} \sin \theta \cos 2 \theta+A_{10} \cos \theta \sin 2 \theta+A_{11} \cos \theta \cos 2 \theta+A_{12} \sin ^{2} 2 \theta \\
& \left.+A_{13} \cos ^{2} 2 \theta\right)
\end{aligned}
$$

where the coefficiens $A_{1}-A_{13}$ are given by:

$$
\begin{gathered}
A_{1}=-\sigma(\dot{l} \cos \alpha-\dot{h} \sin \alpha)+(2 R-\tau)(\dot{l} \sin \alpha+\dot{h} \cos \alpha) \\
A_{2}=\sigma(\dot{l} \cos \alpha-\dot{h} \sin \alpha)+\tau(\dot{l} \sin \alpha+\dot{h} \cos \alpha) \\
A_{3}=\tau(\dot{l} \cos \alpha-\dot{h} \sin \alpha)-\sigma(\dot{l} \sin \alpha+\dot{h} \cos \alpha) \\
A_{4}=-\tau(\dot{l} \cos \alpha-\dot{h} \sin \alpha)+\sigma(\dot{l} \sin \alpha+\dot{h} \cos \alpha) \\
A_{5}=-2 R \sigma \dot{\alpha}-\sigma \dot{\sigma} \\
A_{6}=2 R \sigma \dot{\alpha}-\sigma \dot{\sigma} \\
A_{7}=4 R(\tau-R) \dot{\alpha}+2 R \dot{\sigma} \\
A_{8}=R \sigma \dot{\alpha}+1.5 \sigma \dot{\sigma} \\
A_{9}=-0.5\left(\tau^{2}+\sigma^{2}+2 R \tau\right) \dot{\alpha}-(R \dot{\sigma}+0.5 \tau \dot{\sigma}) \\
A_{10}=0.5\left(\tau^{2}+\sigma^{2}-4 R \tau\right) \dot{\alpha}+0.5 \tau \dot{\sigma} \\
A_{11}=1.5 \sigma \dot{\sigma}-2 R \sigma \dot{\alpha} \\
A_{12}=-0.5 \sigma \dot{\sigma} \\
A_{13}=-0.5 \sigma \dot{\sigma}
\end{gathered}
$$

Using the conjugate function theory[30], the relationship between the circumferential velocity and radial velocity on the circle in the z-plane can be expressed by the integral equation[19]: 


$$
v_{\theta}=-\frac{1}{2 \pi} \int_{0}^{2 \pi} v_{r} \cot \left(\frac{\beta-\theta}{2}\right) d \beta
$$

Substituting the $v_{r}$ into equation (11), we obtain:

$$
\begin{aligned}
& v_{\theta m} \\
& =\frac{1}{R}\left(-A_{1} \cos \theta-\left(A_{2}+0.5 A_{7}\right) \cos 2 \theta+A_{3} \sin \theta+\left(A_{4}-0.5 A_{5}+0.5 A_{6}\right) \sin 2 \theta\right. \\
& -A_{8} \sin \theta \cos 2 \theta+A_{9} \sin \theta \sin 2 \theta-A_{10} \cos \theta \cos 2 \theta+A_{11} \cos \theta \sin 2 \theta-0.5 A_{12} \sin 4 \theta \\
& \left.+0.5 A_{13} \sin 4 \theta+\frac{\Gamma_{m}}{2 \pi}\right)
\end{aligned}
$$

Applying the Kutta-condition at the trailing edge $(\theta=0)$, the bound circulation due to airfoil motion can be obtained as:

$$
\Gamma_{m}=2 \pi\left[2 R(\dot{l} \sin \alpha+\dot{h} \cos \alpha)+\dot{\alpha}\left(\frac{1}{2} \tau^{2}+\frac{1}{2} \sigma^{2}-2 R^{2}\right)+R \dot{\sigma}+0.5 \tau \dot{\sigma}\right]
$$

According to the definition of circulation, the circumferential velocities equals to the linear density of the circulation (or vortex sheet). Thus, the total quasi-steady linear density of the circulation $\left(\gamma_{\theta}\right)$ is obtained by adding up the circumferential velocities due to the freestream and airfoil motion:

$$
\begin{gathered}
\gamma_{\theta}=v_{\theta f}+v_{\theta m} \\
=\frac{1}{R}\left(-A_{1} \cos \theta-\left(A_{2}+0.5 A_{7}\right) \cos 2 \theta+A_{3} \sin \theta+\left(A_{4}-0.5 A_{5}+0.5 A_{6}\right) \sin 2 \theta\right. \\
-A_{8} \sin \theta \cos 2 \theta+A_{9} \sin \theta \sin 2 \theta-A_{10} \cos \theta \cos 2 \theta+A_{11} \cos \theta \sin 2 \theta-0.5 A_{12} \sin 4 \theta \\
\left.+0.5 A_{13} \sin 4 \theta\right)-2 U_{\infty} \sin (\theta-\alpha)+\frac{\Gamma_{0}}{2 \pi R}
\end{gathered}
$$

where the total quasi-steady bound circulation is given by:

$$
\Gamma_{0}=\Gamma_{k}+\Gamma_{m}=2 \pi\left[2 R\left(\left(\dot{l}-U_{\infty}\right) \sin \alpha+\dot{h} \cos \alpha\right)+\dot{\alpha}\left(\frac{1}{2} \tau^{2}+\frac{1}{2} \sigma^{2}-2 R^{2}\right)+R \dot{\sigma}+0.5 \tau \dot{\sigma}\right]
$$

\subsubsection{Unsteady potential solution}

In the z-plane, the wake shedding from the airfoil can be modelled by a series of discrete vortices. For a vortex located at $z_{w}$ with strength $d \Gamma_{w}$, the circle theorem [31] states that the zero-flow-through boundary condition on the circle of radius $R$ is satisfied by placing a vortex inside the circle at $z_{i m}$ with equal strength but opposite sign, as shown in Figure 1. The relation of strength and position of the pair of vortices are given by: 


$$
d \Gamma_{i m}=-d \Gamma_{w} \text { and } z_{i m}=\frac{R^{2}}{\overline{z_{w}}}
$$

Assuming at each time step, the discrete vortices are shed at both leading edge and trailing edge. The induced circumferential velocity on the circle are then obtained by summing over all the shed vortices and bound circulation (17):

$$
v_{\theta u}=-\frac{1}{2 \pi R} \int_{t v} \mathfrak{R}\left(\frac{z_{t v}+R e^{i \theta}}{z_{t v}-R e^{i \theta}}\right) \gamma_{t v} d z_{t v}-\frac{1}{2 \pi R} \int_{l v} \Re\left(\frac{z_{l v}+R e^{i \theta}}{z_{l v}-R e^{i \theta}}\right) \gamma_{l v} d z_{l v}-\frac{\Gamma_{0}}{2 \pi R}
$$

where $\gamma_{t v} d z_{t v}$ and $\gamma_{l v} d z_{t v}$ are trailing edge vortices strength and leading edge vortices strength. The bound circulation $\Gamma_{0}$ is introduced so as to satisfy Kelvin's law that the total circulation within the fluid is zero at each time step.

Applying the Kutta-condition at leading edge and trailing edge, the strength of the latest shed vortices can be solved using the equations (18) and (19):

$$
\begin{gathered}
\Gamma_{0}=-\frac{1}{2 \pi R} \int_{t v} \mathfrak{R}\left(\frac{z_{t v}+R}{z_{t v}-R}\right) \gamma_{t v} d z_{t v}-\frac{1}{2 \pi R} \int_{l v} \mathfrak{R}\left(\frac{z_{l v}+R}{z_{l v}-R}\right) \gamma_{l v} d z_{l v} \\
\frac{1}{R}\left[A_{1}-\left(A_{2}+\frac{1}{2} A_{7}\right)+A_{10}\right]-2 U_{\infty} \sin \alpha=\frac{1}{2 \pi R} \int_{t v} \mathfrak{R}\left(\frac{z_{t v}-R}{z_{t v}+R}\right) \gamma_{t v} d z_{t v}+ \\
\frac{1}{2 \pi R} \int_{l v} \mathfrak{R}\left(\frac{z_{l v}-R}{z_{l v}+R}\right) \gamma_{l v} d z_{l v}
\end{gathered}
$$

where the integral $\frac{1}{2 \pi R} \int_{t v} \Re\left(\frac{z_{t v}+R}{z_{t v}-R}\right) \gamma_{t v} d z_{t v}$ and $-\frac{1}{2 \pi R} \int_{l v} \Re\left(\frac{z_{l v}+R}{z_{l v}-R}\right) \gamma_{l v} d z_{l v}$ are the sum of effects of both previous shed vortices and latest vortices.

The additional linear density of the circulation distributed on the airfoil can be obtained from:

$$
\gamma_{1}=v_{\theta u}=-\frac{1}{2 \pi R} \int_{t v} \Re\left(\frac{z_{t v}+R e^{i \theta}}{z_{t v}-R e^{i \theta}}\right) \gamma_{t v} d z_{t v}-\frac{1}{2 \pi R} \int_{l v} \Re\left(\frac{z_{l v}+R e^{i \theta}}{z_{l v}-R e^{i \theta}}\right) \gamma_{l v} d z_{l v}-\frac{\Gamma_{0}}{2 \pi R}
$$

\subsection{Wake roll-up and collision avoiding mechanism}

In this model, the latest shedding vortices from the leading and trailing edges are placed at $\zeta_{t}=$ $\zeta_{\text {edge }}+\frac{1}{3}\left(-\zeta_{\text {edge }}+\zeta_{t-1}\right)$ in the airfoil-fixed coordinate system [19], as shown in Figure 3. At each time step, the positions of the shed vortices are determined based on the induced velocity caused by all 
other discrete vortices and bound vortices on the airfoil. The convection equation for induced velocity $\bar{w}_{o}$ for a shed vortex at $\zeta_{0}$ in the airfoil fixed coordinate is written as [19]:

$$
\overline{w_{0}}=\overline{W_{\infty}}+\frac{1}{2 \pi i} \int_{\text {bound }} \frac{\left(\gamma_{\theta}+\gamma_{1}\right)}{\zeta_{0}-\zeta} d \zeta+\frac{1}{2 \pi i} \int_{t v} \frac{\gamma_{t v}}{\zeta_{0}-\zeta} d \zeta+\frac{1}{2 \pi i} \int_{l v} \frac{\gamma_{l v}}{\zeta_{0}-\zeta} d \zeta
$$

where $\overline{\mathrm{W}_{\infty}}$ is the conjugate freestream velocity and the three integrals represent the induced velocities of bound vortives, trailing edge vortices and leading edge vortices respectively.

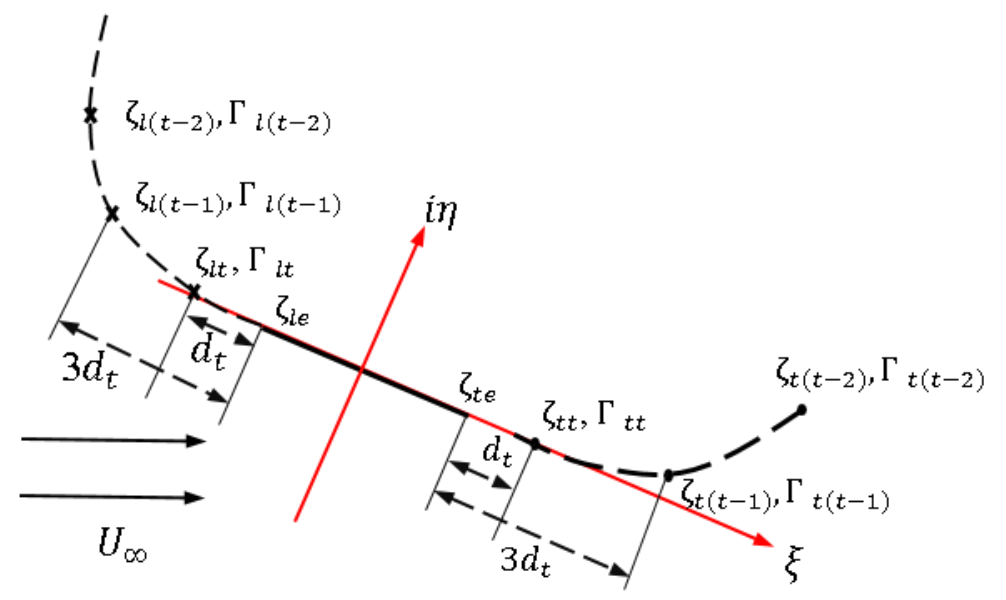

Figure 3. Vortex shedding model for the 2D airfoil

Although the above formalism is sufficed to describe the wake motion on the theoretical basis, certain situations may occur and cause violation of the boundary conditions when implementing numerical analysis in a discrete time marching scheme. This is especially the case for complex wing motions such as flapping wings. For example, consider a wing undergoing only heaving motion in air. As shown in Figure 4, the shed vortices from the leading and trailing edges roll up and stay near the upper surface of the wing in downstroke; when the wing enters upsroke, these vortices on the upper surface would collide with the wing boundary and penetrate to the lower surface. The vortices penetration is because the discrete time marching can't capture the high velocity gradient near the sigular surface of the airfoil boundary. 


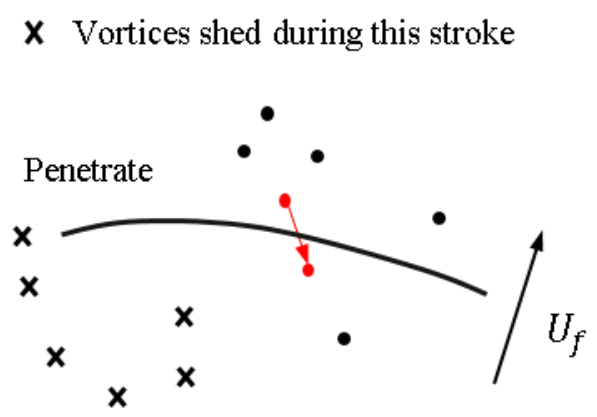

- Vortices shed during previous stroke

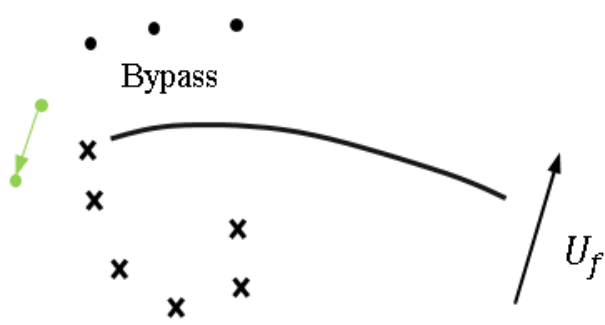

Figure 4. Collision of the vortices with airfoil during vortices convection process

To prevent vortices penetration, an additional cosine type wall function is created and added in the UAM. The wall function is defined in the boundary layer close to the wing surface with distance between $0.02 \mathrm{c}$ to $0.08 \mathrm{c}$ (c denotes the wing chord) in this model, as shown in Figure 5(a). Within this region, the induced velocity $\bar{w}_{o}$ is modified to:

$$
\bar{w}_{o m}=k(d) V_{n} \boldsymbol{n}+V_{t} \boldsymbol{t}
$$

where $k$ is the wall function which takes value between $0 \sim 1 ; d$ is the distance of the vortices from the wing surface; $\boldsymbol{n}$ and $\boldsymbol{t}$ are the unit normal and unit tangential vector field to the wing surface; $V_{n}$ and $V_{t}$ are the normal and tangential component of the induced velocity of the vortices $\bar{w}_{o}$ as expressed in equation (21). In this study, the wall function $k$ is chosen to be continuous function which describes the decay of the normal component of induced velocity $V_{n}$ near the wing surface. Examples of the wall function can be given as cosine function: $k=0.5-0.5 \cos \left[\pi\left(d-B_{l} c\right) /\left(B_{u} c-B_{l} c\right)\right]$, the exponential square function $k=1-e^{-\pi\left(d-B_{l} c\right) /\left(B_{u} c-B_{l} c\right)^{2}}$ and the exponential function $k=$ $1-e^{-\pi\left(d-B_{l} c\right) /\left(B_{u} c-B_{l} c\right)}$, as shown in Figure 5(b). The modified $\bar{w}_{o}$ in the UAM can effectively prevent vortices penetration, but also reduce a little the induced velocities of the vortices near the wing surface in the UAM. As the result, the aerodynamic force calculated by the UAM is normally slightly smaller than the CFD method. 


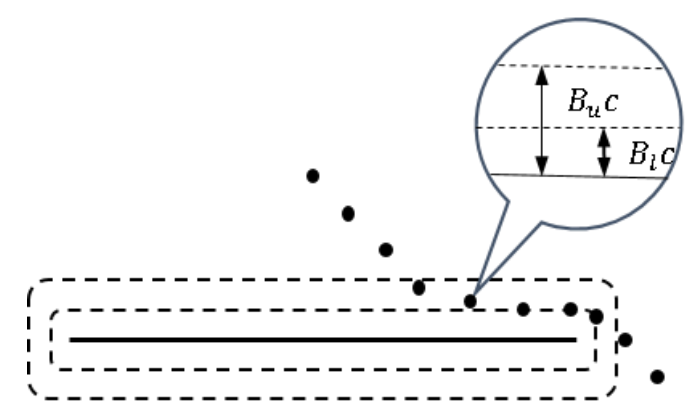

(a) Upper bound and lower bound of wall function

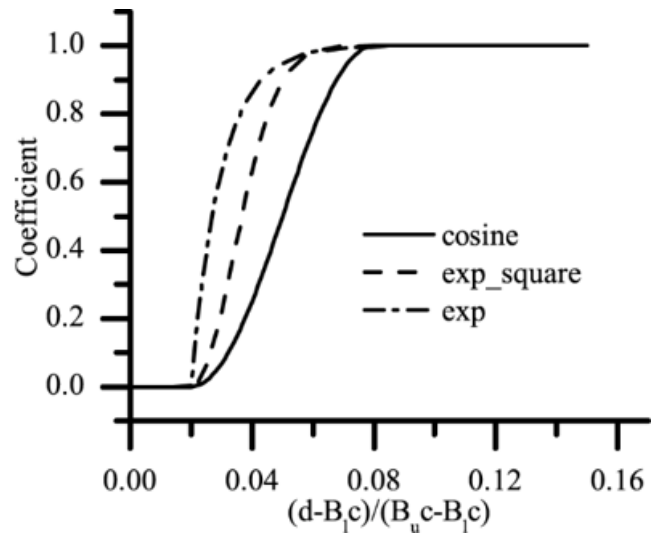

b) Example of wall function

Figure 5. Collision avoiding mechanism for discrete vortices model

\subsection{The extended UAM validation}

In order to validate the extended UAM, two 2D flat plate wings in different Reynolds numbers and two 3D wings have been modelled and compared with previous CFD results in literature. In the first three cases, the wing is assumed to be a rigid plate in prescribed heaving and pitching 'flapping' motion. For the 3D wing, the aerodynamic force along the span is calculated using blade element analysis based on strip theory. In the fourth case, the wing is flexible with an assumed periodic camber variation in a flapping cycle.

Validation case 1 . In this case, a flapping wing of $2 \mathrm{D}$ flat plate is set at $\mathrm{Re}=157$ in the same condition as that by Wang et al [27]. The wing moves in an inclined stroke plane at an angle $\beta=60^{\circ}$ to mimic the kinematic of motion of a dragonfly wing as shown in Figure 6.
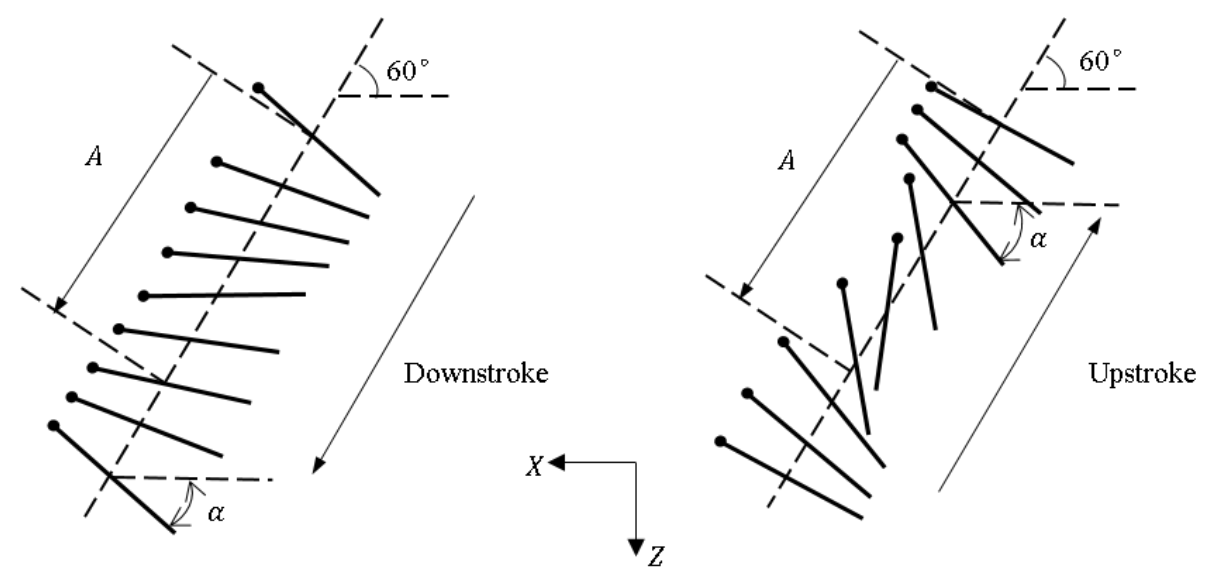

Figure 6. Kinematic of motion of a $2 \mathrm{D}$ flat plate in an inclined stroke plane 
The wing heaving and pitching motion are expressed by the equations:

$$
\begin{aligned}
& A(t)=\frac{A_{0}}{2}\left[-\cos \left(\frac{2 \pi t}{T}\right)+1\right] \\
& \alpha(t)=\alpha_{0}-\frac{\pi}{4} \sin \left(\frac{2 \pi t}{T}+\varphi\right)
\end{aligned}
$$

where $A_{0}$ is the heaving amplitude, $\alpha_{0}$ is the average pitching angle, $T$ is the period of a flapping cycle, $\varphi$ is the phase difference between pitching and heaving motion.

The wing motion in displacement and pitching angle in two flapping cycles are shown in Figure 7. $X$ and $Z$ denotes the forward and downward flapping displacement respectively. Based on the prescribed kinematics of motion, the instantaneous forces from the UAM reached stable periodical oscillation after five flapping cycles. The comparisons of the aerodynamic lift and drag coefficient results by the UAM and previous literature results using CFD method by Kim and Choi, Wang [27,28] are shown in Figure 8.

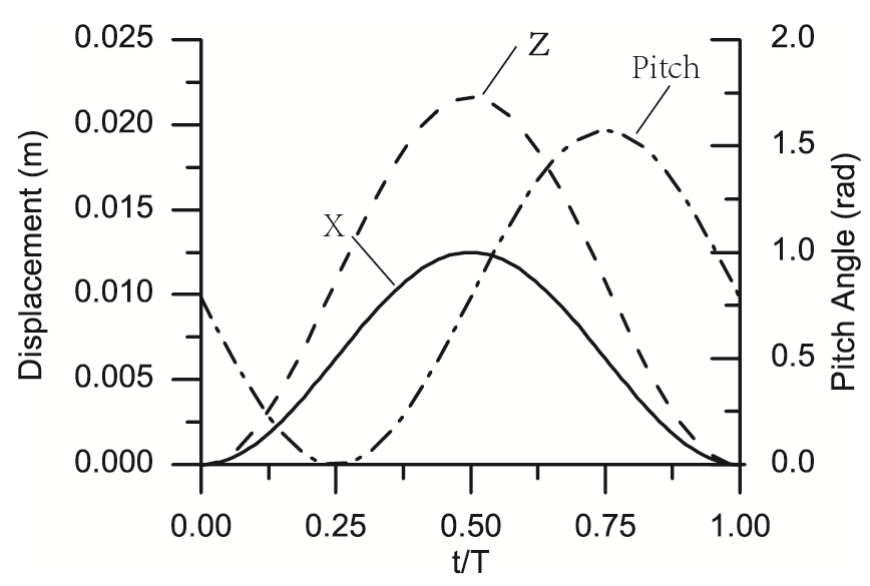

Figure 7. The position and pitching angle of the flapping wing in a cycle of flapping amplitude. $A_{0}=25 \mathrm{~mm}$, flapping period $\mathrm{T}=0.025 \mathrm{~s}$, stroke plane inclination angle $\beta=\frac{\pi}{3} \mathrm{rad}$, phase difference $\varphi=0$ rad, average pitching angle $\alpha_{0}=\frac{\pi}{4}$. 

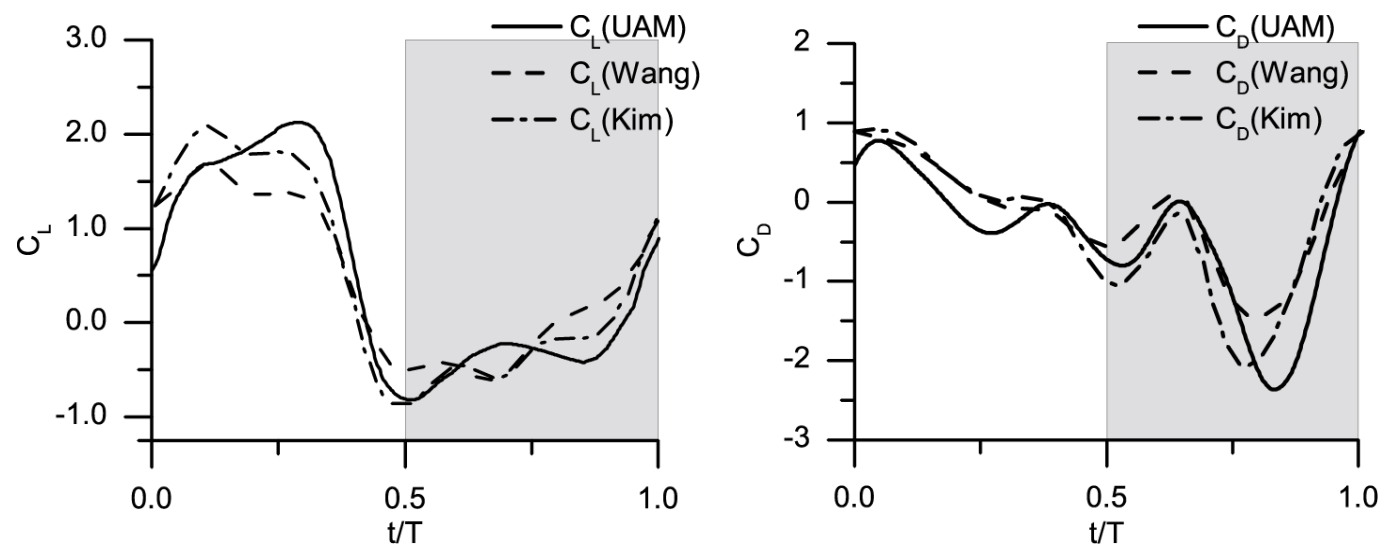

Figure 8. Lift coefficient and drag coefficient in one flapping cycle

As shown in Figure 8, the variation of the lift and drag coefficients from the UAM is of similar trend to the CFD results by Kim and Choi[28], and Wang [27]. The peak values agree very well in both downstroke and upstroke although the $\mathrm{C}_{\mathrm{L}}$ peak timing from the UAM in downstroke is slightly later than that from the CFD method. This timing delay is mainly caused by the influence of fluid viscosity especially at low Reynolds number not being included in the UAM based on potential flow theory [32]. The mean lift coefficient $\mathrm{C}_{\mathrm{L}}=0.487$ in a flapping cycle calculated from the UAM is close to the CFD result $C_{L}=0.49$ obtained by Wang [27] but a larger difference is noted from $C_{L}=0.42$ by Kim and Choi [28]. The variation of drag coefficient in a flapping cycle from the UAM also shows similar trend to the CFD results by Kim's and Wang. Although the average drag coefficient $C_{D}=-0.43$ from the UAM is reasonably close to the CFD result $C_{D}=-0.5$ by Kim and Choi, there is a considerably large difference from another $C F D$ result $C_{D}=-0.28$ by Wang.

Figure 9 shows the evolution of the flow field in a flapping cycle from the UAM, where red dots and blue dots indicate clockwise and anti-clockwise rotating vortices respectively. It is noticed that the leading edge vortices (LEV) and trailing edge vortices (TEV) are shed in rotating pairs at each time step. In the downstroke, the LEV and TEV rotate in clockwise and anti-clockwise direction respectively; in the upstroke, the LEV and TEV change rotation direction. 

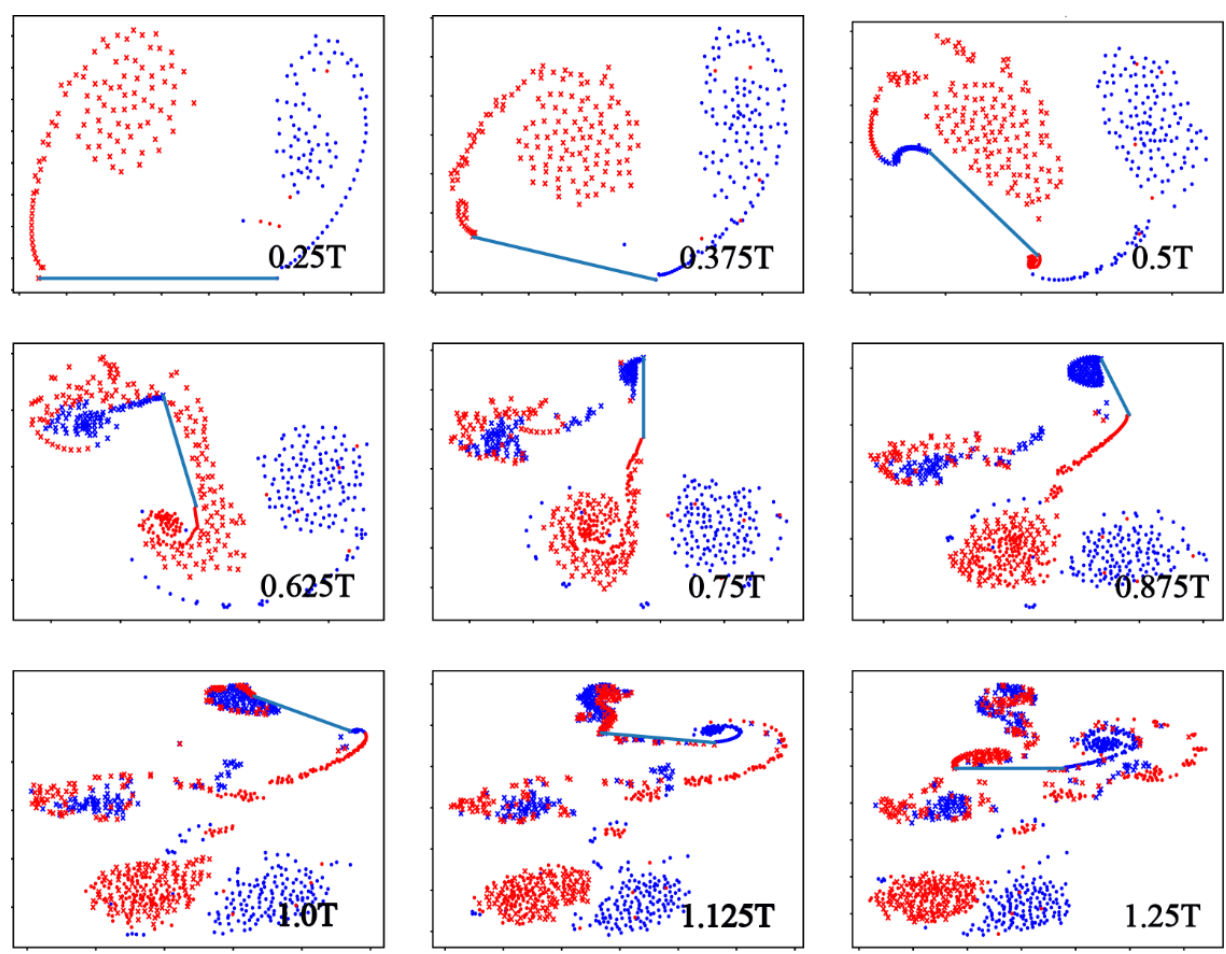

Figure 9. Snapshots of flow field in one flapping period (blue dots indicates anti-clockwise rotating vortices and red dots indicate clockwise rotating vortices)

Validation case 2. In this case, a 2D flat plate of chord length $10 \mathrm{~mm}$ is set in horizontal flapping motion in the same condition as that by Wang et al [7]. The heaving and pitching motion function are defined in equation ( 23 ) and (24) with an average pitching angle $\alpha_{0}=\frac{\pi}{4}$, heaving amplitude $A_{0}=28 \mathrm{~mm}$ and flapping period T $=4 \mathrm{~s}$.

The resulting lift coefficients in this case are shown in Figure 10 in comparison with the CFD and experimental result by Wang et al [7]. According to Figure 10, the lift coefficient curve from the UAM shows the same trend as the CFD and experimental result. It is noted that there are two lift coefficient peaks in downstroke and another two peaks in upstroke. During each of the half stroke, the first peak occurs at about $t / T=0.1$ and $t / T=0.6$ while the second one occurs at about $t / T=0.3$ and $t / T=$ 0.8. The amplitudes of the peaks from the UAM are close to that by Wang et al[7]. The average lift coefficient $\mathrm{C}_{\mathrm{L}}=0.468$ obtained from the UAM is only $1.8 \%$ and $5 \%$ smaller than the CFD result $C_{L}=0.479$ and the experimental result $C_{L}=0.494$. 


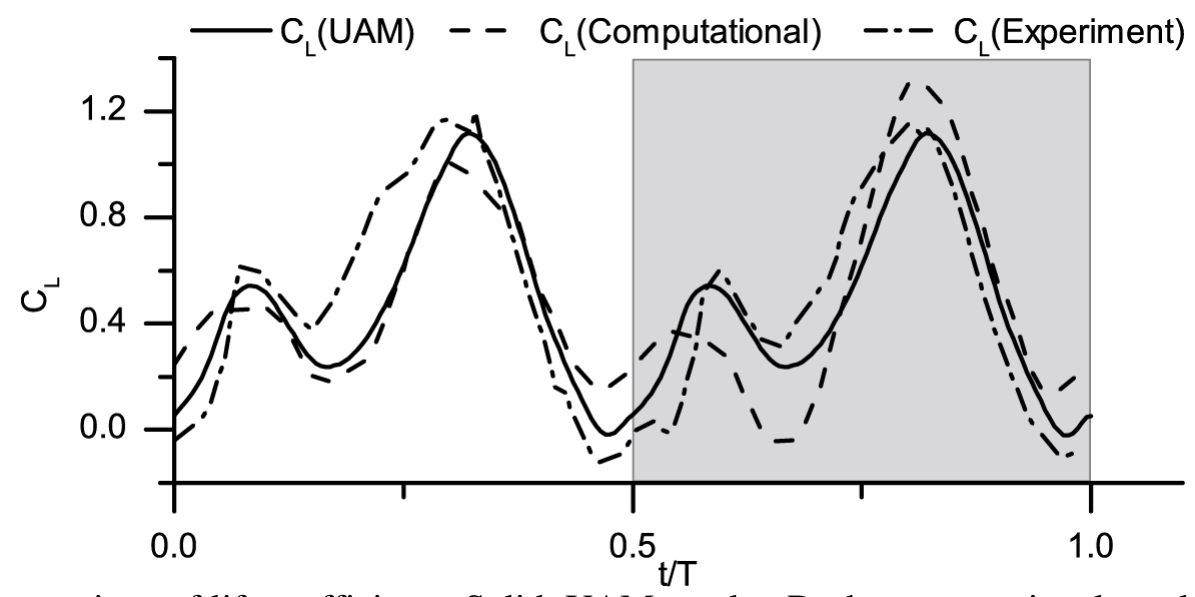

Figure 10. Comparison of lift coefficients. Solid, UAM results; Dash, computational results by Wang et al; Dash dot, experimental data by Wang et al.

Validation case 3. To further validate the UAM, a 3D wing model is taken with the same elliptical platform at $\mathrm{Re}=1800$ as defined in previous study by $\mathrm{Wu}$ and $\mathrm{Sun}[29]$. In this case, the UAM for a 2D wing is extended to calculate the aerodynamic force of the 3D wing based on strip theory (16 segments). In order to take the 3D effect into account, e.g. spanwise flow and wingtip vortices, an elliptical distribution of spanwise aerodynamic force is selected.

Table 1. Geometric data and kinematic parameters for the 3D wing model

\begin{tabular}{ccccccccc}
\hline $\boldsymbol{B}(\mathbf{m})$ & $\boldsymbol{\Phi}(\mathbf{d e g})$ & $\boldsymbol{\tau}_{\boldsymbol{c}}$ & $\widetilde{\boldsymbol{\omega}}$ & $\boldsymbol{U}_{\boldsymbol{r e f}}(\mathbf{m} / \mathbf{s})$ & $\boldsymbol{\Delta} \boldsymbol{\varphi ( \mathbf { d e g } )}$ & $\boldsymbol{n}_{\boldsymbol{f}}$ & $\boldsymbol{n}_{\boldsymbol{r}}$ & $\boldsymbol{\varphi}_{\boldsymbol{m}}(\mathbf{d e g})$ \\
\hline 0.1015 & 150 & 9.37 & 0.93 & 0.874 & 50 & 2.5 & 12.5 & 40 \\
\hline
\end{tabular}

In Table $1, B$ is the wing semi-span length; $\tau_{c}$ is related to $\Phi$ by $\tau_{c}=2 \Phi\left(\frac{r_{2}}{B}\right)\left(\frac{B}{C}\right)$ where $r_{2}$ is the radius of second moment of wing area $\left(r_{2} / R \approx 0.6\right) ; C$ is chord length. $\widetilde{\omega}$ is a non-dimensional angular velocity of rotation; $U_{r e f}$ is the reference velocity defined by the average flapping velocity at $r_{2} ; \Delta \varphi$ is the amplitude of rotation as shown in Figure 11(b) [29]. $n_{f}$ and $n_{r}$ are stroke frequency and rotation frequency, respectively; $\Phi$ is stroke amplitude; $\varphi_{m}$ is the angle of attack of the wing at mid-stroke. The flapping translational velocity at the span location $r_{2}$ and angular velocity of rotation is expressed by the following equations respectively:

$$
u_{t}=0.5 \pi U_{r e f} \sin \left(\frac{2 \pi t U_{r e f}}{\tau_{c} c}\right)
$$




$$
\omega=\left\{\begin{array}{lc}
\frac{U_{r e f}}{c} \widetilde{\omega}\left[-1-\cos \left(2 \pi \frac{n_{r}}{n_{f}} \hat{t}\right)\right] & 0<\hat{t} \leq 0.1 \text { or } 0.9<\hat{t} \leq 1 \\
\frac{U_{r e f}}{c} \widetilde{\omega}\left[1-\cos \left(2 \pi \frac{n_{r}}{n_{f}} \hat{t}\right)\right] & 0.4<\hat{t} \leq 0.6
\end{array}\right.
$$

where $\hat{t}=t \cdot n_{f}-I N T\left(t \cdot n_{f}\right)$ and $t$ stands for the time. The flapping and pitching motion of the wing in a horizontal plan as defined above is illustrated in Figure 11.

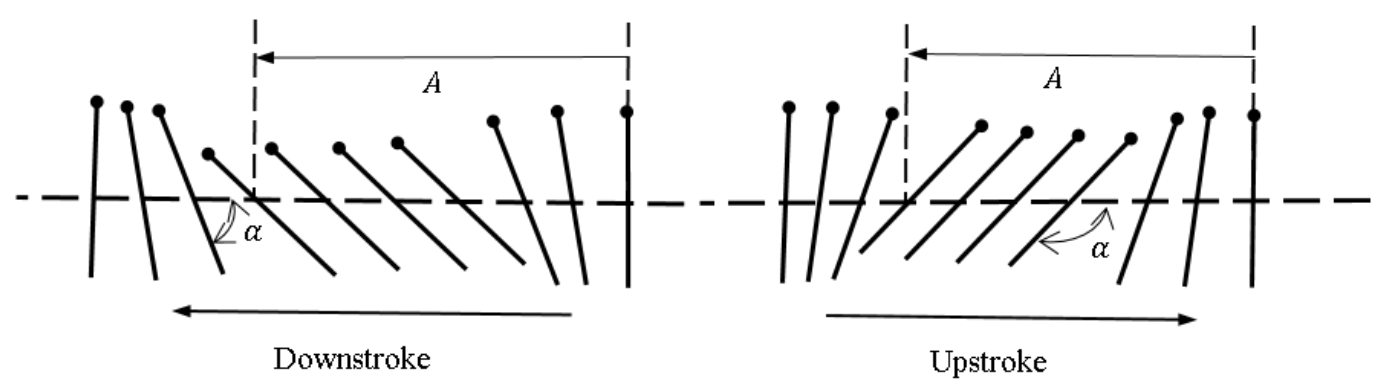

(a) The position of horizontal flapping wing in one cycle

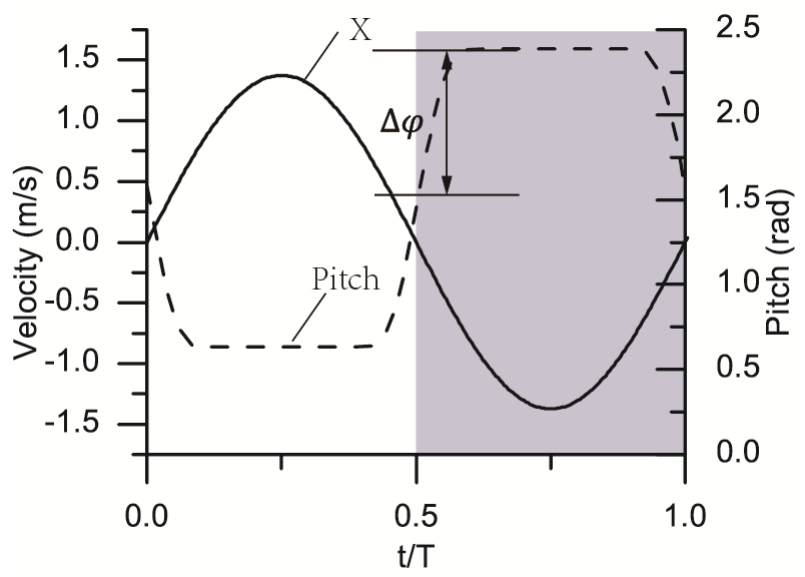

(b) Angular velocity and pitching angle in one cycle and Flapping velocity at span location $r_{2}$

Figure 11. Flapping and pitching motion of the wing in a horizontal plan

As shown in Figure 12, the lift coefficient curve and drag coefficient curve from the UAM show similar trend and close peak values to the CFD results by $\mathrm{Wu}$ and Sun [29] although there is a clear timing difference. The lift coefficient peak from the UAM at $t / T=0.3$ occurs later than the CFD result $(t / T=0.25)$, while the drag coefficient peak at $t / T=0.2$ occurs earlier than the CFD result $(t / T=0.28)$ 

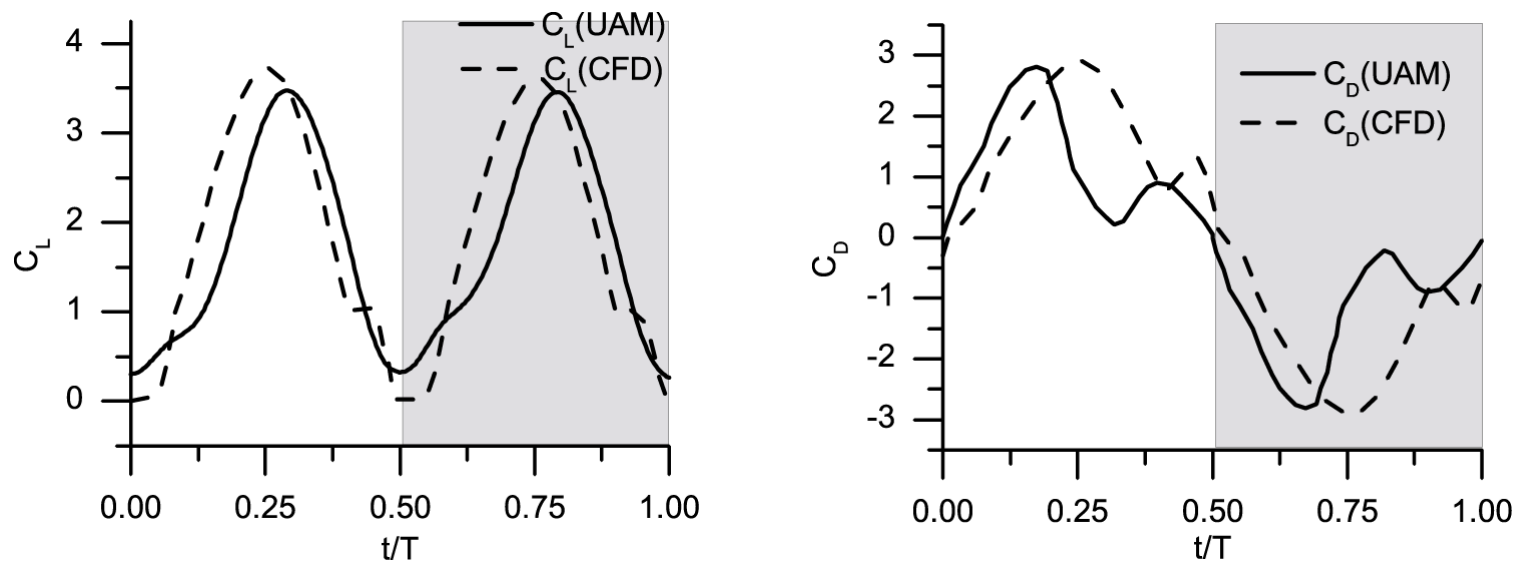

Figure 12. Comparison of lift and drag coefficients of 3D rigid wing by UAM and CFD

Similar phase difference of lift and drag coefficients can also be found in horizontal flapping cases between a 2D airfoil CFD results by Wang et al[7](shown in validation case 2) and 3D wing CFD results by $\mathrm{Wu}$ and Sun[29](shown in validation case 3). It is because the 3D flow included in the CFD models affects the spanwise vortices distribution while the 2D based UAM could not take the 3D flow into account. However, the 3D flow does not have significant effect on the average lift coefficient in a flapping cycle. In this case, the mean lift coefficient of a flapping cycle from the UAM $\left(C_{L}=1.72\right)$ is slightly greater than the CFD result $\left(\mathrm{C}_{\mathrm{L}}=1.70\right)$ by only $1.17 \%$. However the average drag coefficient $C_{D}=1.20$ from the UAM in downstroke is $16 \%$ smaller than the CFD result $C_{D}=1.43$.

Validation case 4. In this case, a 3D wing of variable camber at $\mathrm{Re}=200$ in flapping motion is set in the same condition as that by Du and Sun [22]. The wing shape keeps the same as case 3, but scaled down in dimension to match $\mathrm{Re}=200$. The detailed geometric data and kinematic parameters of this wing model are listed in Table 2.

Table 2. Geometric data and kinematic parameters of the 3D cambering wing

\begin{tabular}{ccccccccc}
\hline $\boldsymbol{B}(\mathbf{m})$ & $\boldsymbol{\Phi}(\mathbf{d e g})$ & $\boldsymbol{\tau}_{\boldsymbol{c}}$ & $\widetilde{\boldsymbol{\omega}}$ & $\boldsymbol{U}_{\boldsymbol{r e f}}(\mathbf{m} / \mathbf{s})$ & $\boldsymbol{\Delta} \boldsymbol{\varphi ( \mathbf { d e g } )}$ & $\boldsymbol{n}_{\boldsymbol{f}}$ & $\boldsymbol{n}_{\boldsymbol{r}}$ & $\boldsymbol{\varphi}_{\boldsymbol{m}}(\mathbf{d e g})$ \\
\hline 0.0371 & 150 & 9.37 & 0.93 & 0.291 & 50 & 2.5 & 12.5 & 40 \\
\hline
\end{tabular}

The camber variation of the wing during the flapping motion is defined in the following function: 


$$
m(\hat{t})= \begin{cases}m_{0}\left\{\left[\frac{2(\hat{t}-0.4)}{0.2}\right]-1\right\} & 0.0 \leq \hat{t}<0.1 \\ m_{0} & 0.1 \leq \hat{t}<0.4 \\ m_{0}\left\{1-\left[\frac{2(\hat{t}-0.4)}{0.2}\right]\right\} & 0.4 \leq \hat{t}<0.6 \\ -m_{0} & 0.6 \leq \hat{t}<0.9 \\ m_{0}\left\{\left[\frac{2(\hat{t}-0.4)}{0.2}\right]-1\right\} & 0.9 \leq \hat{t}<1.0\end{cases}
$$

where $m_{0}$ is the maximum wing $\operatorname{camber}\left(\approx \frac{5 \sigma}{4 c}\right)$, which is $6 \%$ in this case. $\hat{t}=t \cdot n_{f}-I N T\left(t \cdot n_{f}\right)$ and $t$ stands for the time. The comparison of lift and drag coefficients of this case by UAM and CFD results from Du and Sun [22] are shown in Figure 13.

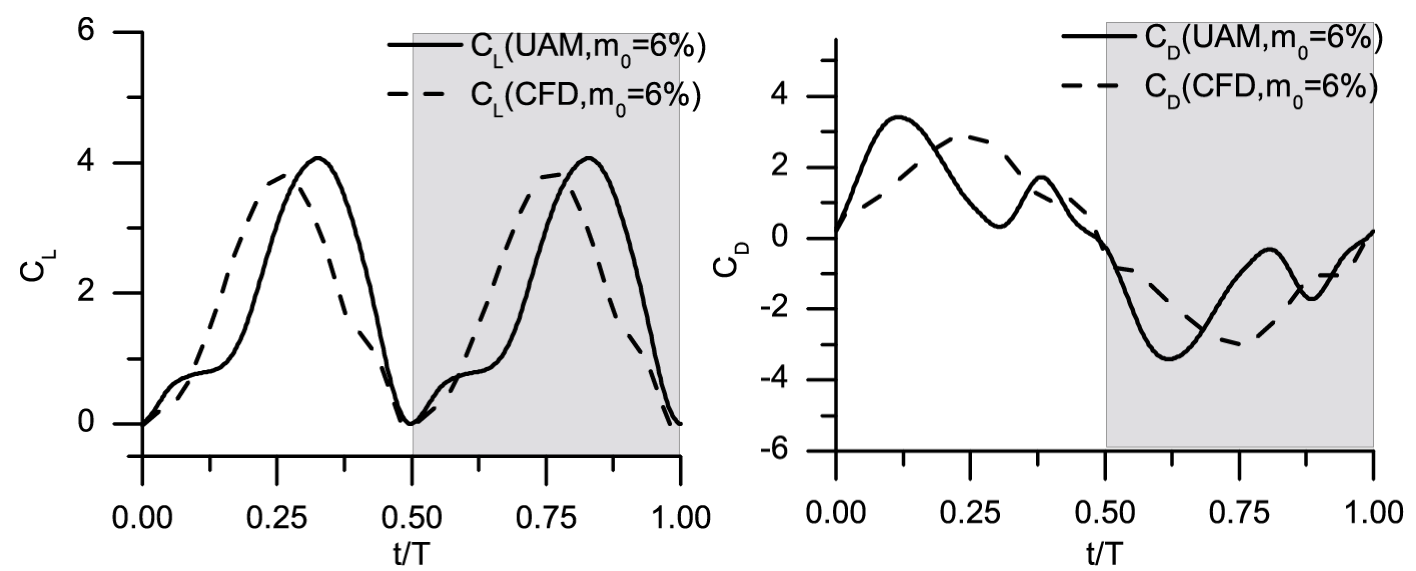

Figure 13. The lift and drag coefficients from UAM and CFD of the 3D wing with variable camber

As shown in Figure 13, the time courses of the lift and drag coefficients of the wing with variable camber from the UAM agree with CFD results in their variation trend despite the same phase differences as given in validation case 3 between these curves still exist. The lift coefficient curve by UAM reaches peak later than that by CFD while the drag coefficient curve by UAM reaches peak earlier than that by CFD. The mean lift coefficient $\mathrm{C}_{\mathrm{L}}=1.86$ from the UAM is in close agreement $(2.8 \%$ difference) with the $C_{L}=1.81$ from $C F D$ result [22]. The mean drag coefficients $C_{D}=1.52$ from the UAM during the downstroke is less than $10 \%$ different from the CFD result $C_{D}=1.68$.

\section{Camber Variation and Results}

For a typical vertical or inclined flapping wing such as dragonfly wing, apparent deformation can be observed during the flapping process. Attention is therefore paid to the aerodynamic performance of a 
vertically flapping wing with camber variation. Firstly a $2 \mathrm{D}$ wing with a prescribed asymmetric camber variation in up and downstroke of a flapping cycle is considered. The wing remains flat in downstroke, and changes to a positive camber in upstroke. Secondly, the study is extended to a 3D wing of variable camber due to structure flexibility to evaluate aeroelastic coupling effect. The instantaneous deformation of the flapping wing subjected to aerodynamic forces is calculated using Abaqus package based on finite element method interfacing with the UAM code at each time step of the simulation process. The aerodynamic results are compared with the rigid wing of the same dimension and flapping motion.

\subsection{D flapping wing of variable camber}

In this case, the 2D wing has a variable camber during upstroke only but keep rigid in downstroke. The wing motion can be expressed as follows with maximum flapping amplitude $\varphi_{\max }$ and pitching angle $\alpha_{\max }$ at mid-stroke.

$$
\begin{array}{r}
\varphi=\varphi_{\text {max }} \cos (2 \pi \hat{t}) \\
\alpha=\alpha_{\text {max }} \sin (2 \pi \hat{t})
\end{array}
$$

where $\hat{t}=t \cdot n-I N T(t \cdot n)$ and $t$ stands for the time; $n$ is the flapping frequency. The continuous change of the wing camber from zero (flat) in the downstroke to the maximum $\left(m_{0}\right)$ in the midupstroke in a flapping cycle is written in the following function and illustrated in Figure 14:

$$
m(\hat{t})=\left\{\begin{array}{lc}
0 & 0.0 \leq \hat{t}<0.5 \\
m_{0}[1-4 \cdot(0.75-\hat{t})] & 0.5 \leq \hat{t}<0.75 \\
m_{0}[1-4 \cdot(\hat{t}-0.75)] & 0.75 \leq \hat{t}<1.0
\end{array}\right.
$$



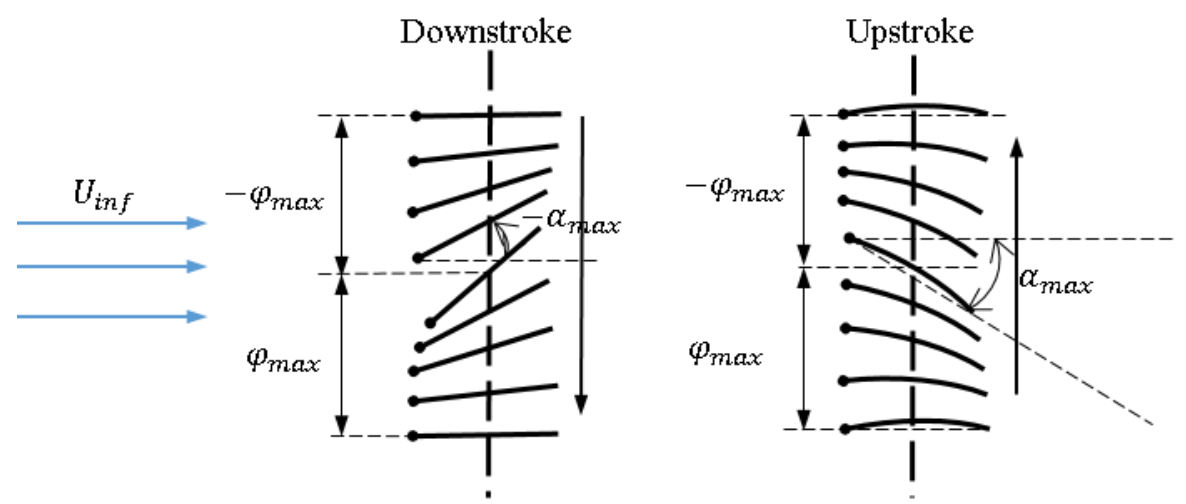

Figure 14. Kinematics of motion of a $2 \mathrm{D}$ wing with asymmetric camber (positive camber in upstroke) A case study is carried out to evaluate the effect of wing camber combined with pitching angles on the aerodynamic performance of the flapping wing. There are four wing camber $m_{0}=0 \%, 3 \%, 6 \%$ and $9 \%$ and two pitching angles $\alpha_{\max }=35^{\circ}$ and $45^{\circ}$ included in the study. The wing chord length is $0.05 \mathrm{~m}$ at $\mathrm{Re}=5400$. Table 3 shows the kinematic parameters for the wing flapping and pitching motion.

Table 3. Kinematic parameters of the 2D flapping wing

\begin{tabular}{cccc}
\hline$\varphi_{\max }(\mathrm{m})$ & $n(\mathrm{~Hz})$ & $\alpha_{\max }(\mathrm{deg})$ & $U_{\text {inf }}(\mathrm{m} / \mathrm{s})$ \\
\hline 0.04 & 10 & 35 and 45 & 1.25 \\
\hline
\end{tabular}

In this study, the commercial solver Fluent is employed to perform the CFD simulation. As shown in Figure 15(a), the fluid field is divided into inner-fluid and outer-fluid field. The inner-fluid field flaps and rotates with the airfoil and will not be reconstructed during the calculation process. It will prevent the mesh near the airfoil surface becoming distorted. If the mesh near the airfoil surface is over skewness, the calculation will terminate to avoid low mesh quality. The radius of interior circle is twice the chord length; the radius of the far field circle is 10 times the chord length. In order to fulfill the rigid motion and camber variation, three functions are built in the user-defined functions (UDF) scripts. The NACA4Digits function is used to define standard NACA airfoil while DEFINE_CG_MOTION and DEFINE_GRID_MOTION are used to define rigid wing motion and variable camber wing motion respectively as shown in Figure 15(b). 


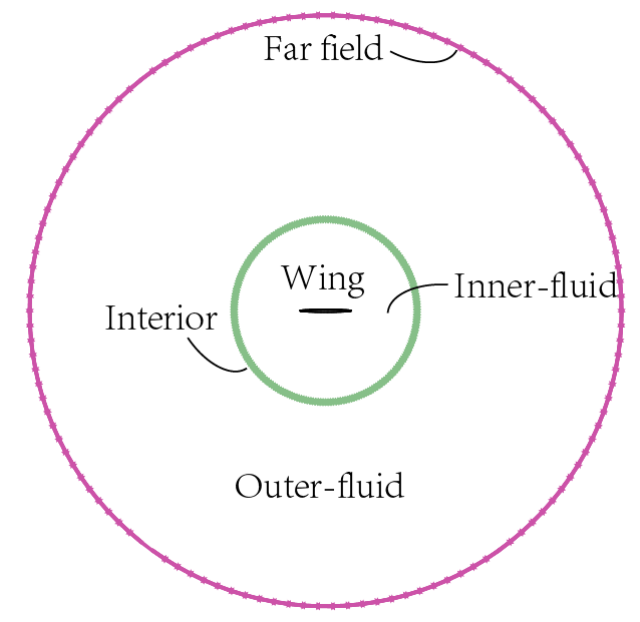

(a)
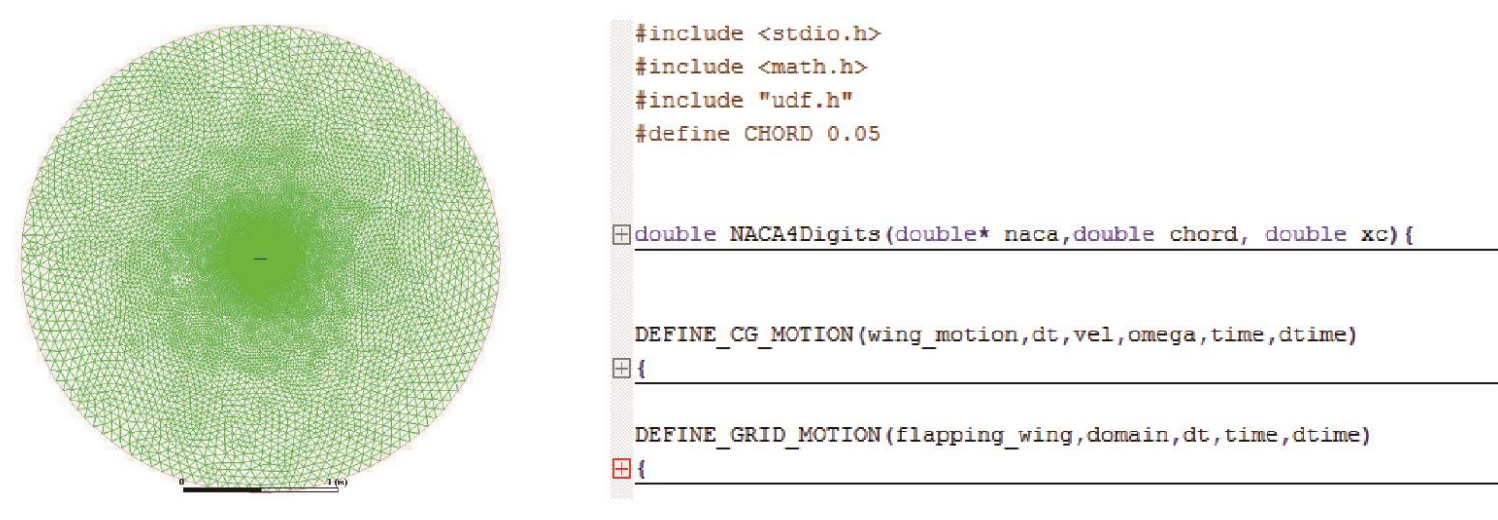

(b)

Figure 15. (a) Diagram of the CFD model (b) Mesh and UDF scripts of the CFD model

The results show that for both rigid $\left(m_{0}=0\right)$ and variable camber $\left(m_{0}>0\right)$ wings in downstroke, the mean lift coefficient $\bar{C}_{L}$ calculated using the UAM and CFD method have negligible small difference. The $\bar{C}_{L}$ in upstroke and a whole flapping cycle varies with the camber and pitching angles as shown in Figure 16(a) and (b). It is noted that the $\bar{C}_{L}$ from the UAM has change rate close to CFD results consistently although the absolute value is slightly smaller than the CFD. The $\bar{C}_{L}$ values from both methods increase with the camber $m_{0}$ and the wing pitching angle $\alpha_{\max }$. This is mainly due to the increase of the effective angle of attack (AoA) in the upstroke and consequently reduced negative lift on the wing as shown in (c) 
Figure 16(a). Taking the case of $m_{0}=9 \%$ and $\alpha_{\max }=35^{\circ}$ for further analysis, the negative $\bar{C}_{L}=-$ 0.689 from the UAM is reduced by $22 \%$ due to the positive camber comparing with the $\bar{C}_{L}=-0.882$ of the rigid wing $\left(m_{0}=0\right)$ in upstroke; similarly the resulting negative $\bar{C}_{L}=-0.783$ from the wing of positive camber is reduced by $20.7 \%$ comparing with the $\bar{C}_{L}=-0.988$ of the rigid wing in upstroke by using the CFD method. For a complete flapping cycle, the results as shown in (c)

Figure 16(b) indicate that the $\bar{C}_{L}$ of the camber wing is increased significantly compared with the rigid one. As shown in (c)

Figure 16(c), the time courses of the $C_{L}$ in a flapping cycle obtained by both methods have similar trend for both the rigid wing and camber wing $\left(m_{0}=9 \%\right)$. In the downstroke $\mathrm{t} / \mathrm{T}=0 \sim 0.5$, the results by using either the UAM or the CFD method keep virtually the same as a rigid flat wing since the wing has zero camber. In the upstroke $\mathrm{t} / \mathrm{T}=0.5 \sim 1$ however, the positive camber leads to a reduced negative $C_{L}$ comparing with the rigid wing.

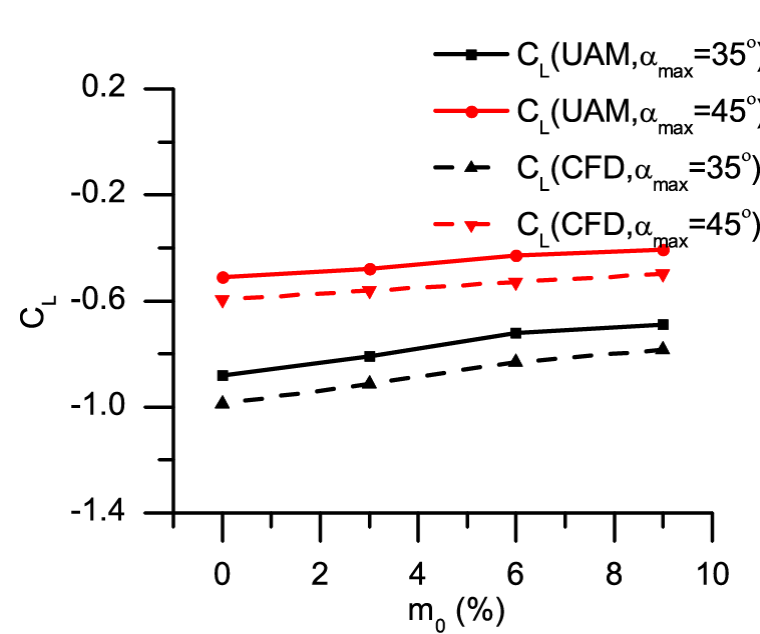

(a)

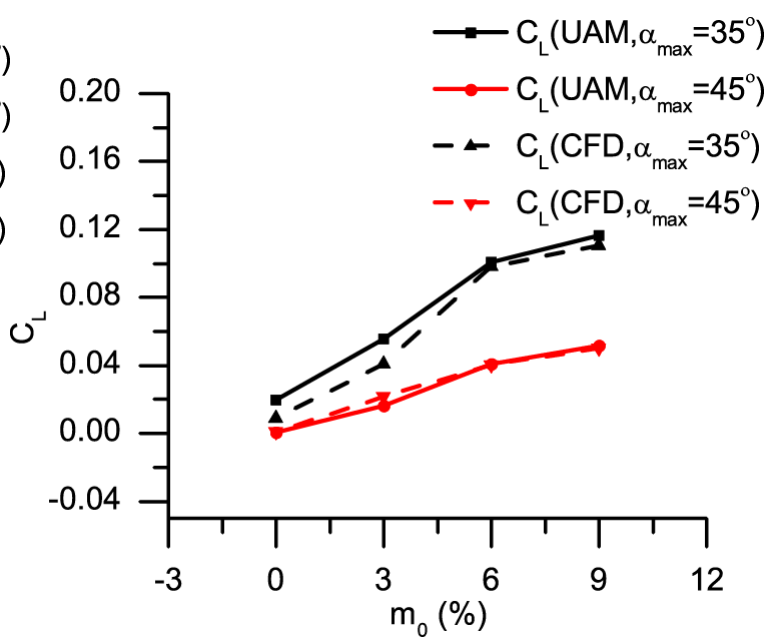

(b) 


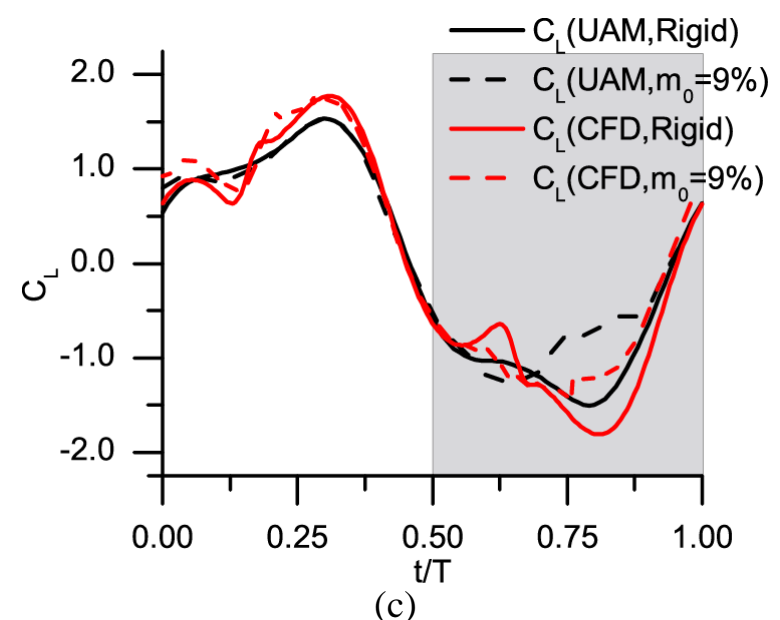

Figure 16. (a) Mean $C_{L}$ in upstroke only (b) mean $C_{L}$ of a flapping cycle varying with pitching angle $\left(\alpha_{\max }\right)$ and camber $\left(m_{0}\right)(\mathrm{c})$ instantaneous $\mathrm{C}_{\mathrm{L}}$ of the rigid and camber wing $\left(m_{0}=9 \%, \alpha_{\max }=35^{\circ}\right)$

The study results imply that a flapping wing of asymmetric structure bending stiffness would improve the aerodynamic performance. Such a flexible wing structure can be designed by imposing a mechanical constraint to the wing bending in downstroke and allowing for adaptive bending to gain a positive camber in downstroke[23].

\subsection{Aeroelastic effect on a flexible flapping $3 D$ wing}

In this final case study, a flexible 3D wing flapping in a prescribed vertical and pitching motion is considered. The prescribed motion of the wing is the same as the $2 \mathrm{D}$ wing expressed in equations and (29) except that the flapping amplitude varies along the span with a different maximum pitching angle.

$$
\begin{gathered}
\phi=0.5 \cdot \phi_{0} \cos (2 \pi \hat{t}) \\
\alpha=\alpha_{\max } \sin (2 \pi \hat{t})
\end{gathered}
$$

where $\hat{t}$ is the non-dimensional time scaled by the flapping period; $\phi$ is the flapping angle; $\phi_{0}$ is the flapping angle amplitude; $\alpha$ is the pitching angle of the wing; $\alpha_{\max }$ is the maximum pitching angle at mid-stroke. In this case, $\phi_{0}=\frac{\pi}{3} \mathrm{rad}, \alpha_{\max }=-\frac{\pi}{6} \mathrm{rad}$, the flapping frequency $10 \mathrm{~Hz}$ and $\mathrm{Re}=7800$. The wing is a flat rectangular platform of $100 \mathrm{~mm}$ semi-span and $50 \mathrm{~mm}$ chord length in flapping motion as illustrated in Figure 17. 


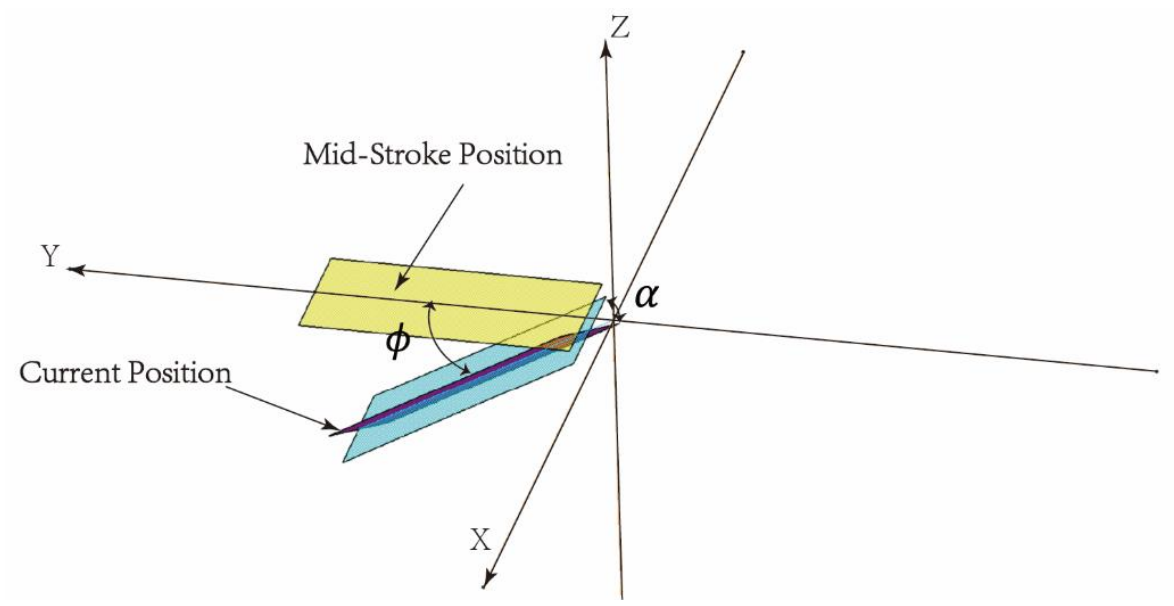

Figure 17. Illustration of the 3D flapping wing motion

The wing is constructed with three beams of rectangular cross section set in different orientations and connected at the wing root and covered by a membrane skin as illustrated in Figure 18. The material properties of the carbon/epoxy beams and the capran film skin as defined in [34] are listed in Table 4. By employing finite element (FE) method, the skin is modelled using shell element and the beams modelled using beam element.

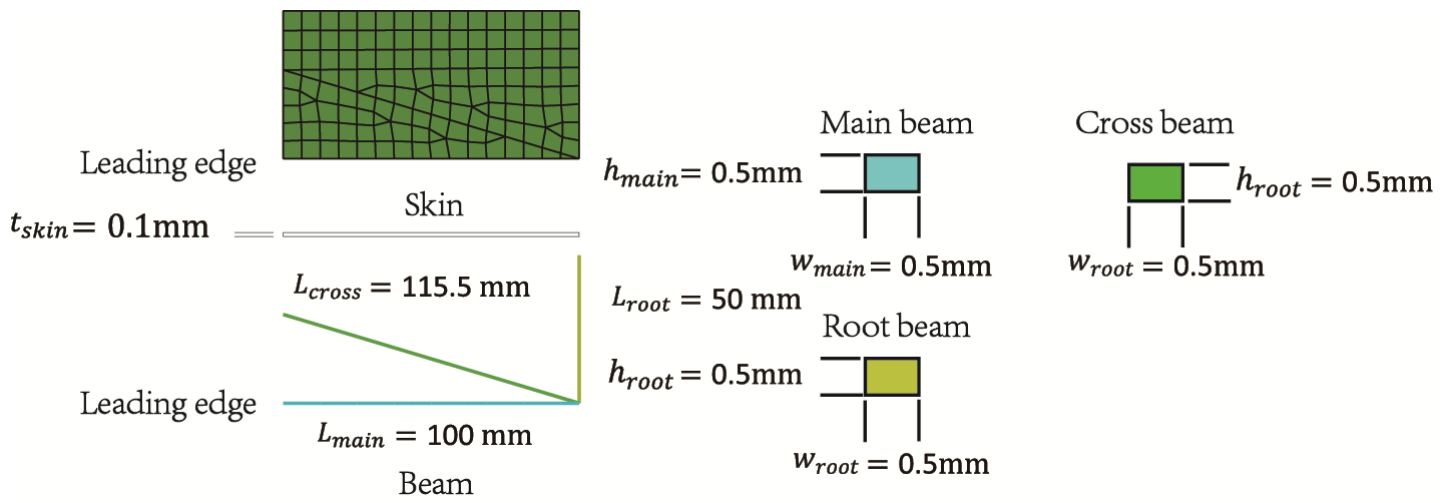

Figure 18. The wing structure configuration and components FE models

Table 4. Material properties of the wing structure components

\begin{tabular}{ccccccc}
\hline $\begin{array}{c}\boldsymbol{E}_{\text {beam11 }} \\
(\boldsymbol{G P a})\end{array}$ & $\begin{array}{c}\boldsymbol{E}_{\text {skin }} \\
(\boldsymbol{G P a})\end{array}$ & $\begin{array}{c}\boldsymbol{E}_{\text {beam12 }} \\
(\boldsymbol{G P a})\end{array}$ & $\begin{array}{c}\boldsymbol{E}_{\text {beam22 }} \\
(\boldsymbol{G P a})\end{array}$ & $\boldsymbol{v}_{\text {beam12 }}$ & $\begin{array}{c}\boldsymbol{\rho}_{\text {beam }} \\
\left(\mathrm{kg} / \mathbf{m}^{3}\right)\end{array}$ & $\begin{array}{c}\boldsymbol{\rho}_{\text {skin }} \\
\left(\mathrm{kg} / \mathbf{m}^{\mathbf{3}}\right)\end{array}$ \\
\hline 233 & 3 & 3 & 23.1 & 0.05 & 1740 & 1160 \\
\hline
\end{tabular}

In the structural analysis, the elastic deformation of the 3D wing is calculated using the FE-based Abaqus software interfacing with the UAM code in a strong coupling approach. The wing surface is evenly divided into 8 sections along the span as shown in Figure 19(a). The aerodynamic forces will 
not change significantly if more sections are selected with increasing computing time. At each time step of simulation in a flapping cycle, the instantaneous shape and velocity of the wing are fed into the UAM to calculate the unsteady aerodynamic forces. As a result of aeroelastic coupling due to the interaction between the inertial, elastic and aerodynamic forces, the wing deforms continuously with each spanwise section decomposed into camber and twist at the following time step. As illustrated in Figure 19(b), the camber is obtained by curve fitting of the leading edge $P_{1}$, mid-chord $P_{2}$ and trailing edge $P_{3}$ of the wing section to determine the ratio of the distance of the circle arc between $P_{1}$ and $P_{3}$ to the undeformed chord length. The twist angle is calculated from the angle between the chord lines of the deformed $P_{1}$ and $P_{3}$ and undeformed position.

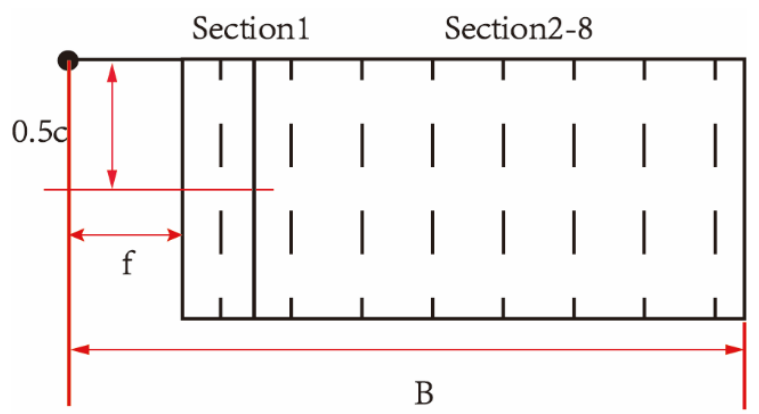

(a)

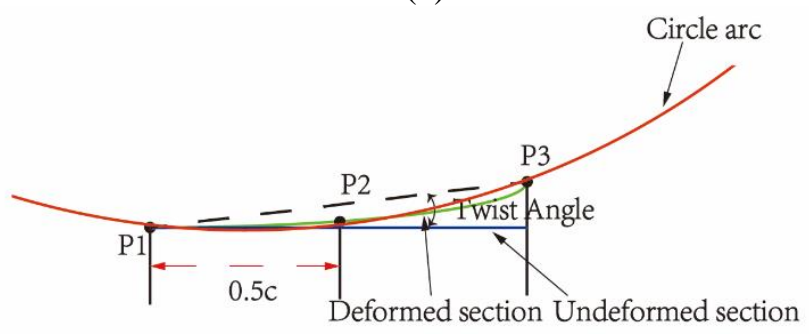

(b)

Figure 19. (a) The wing spanwise sections (b) measurement of a wing section camber and twist angle From the analysis, the resulting time courses of the wing camber variation of a few spanwise sections in a flapping cycle are shown in Figure 20(a). It is noted that despite small deformation of the membrane skin, the wing deforms in negative camber in downstroke and positive camber in upstroke with the value increasing from the wing root to tip. In downstroke, the aerodynamic lift would cause a negative camber since the cross beam and the unreinforced trailing edge of the wing will deflect up relative to the leading edge. It is also noted that the camber of all the wing sections changes sign at 
about $\mathrm{t}=0.4 \mathrm{~T}$ and $0.9 \mathrm{~T}$ in a flapping cycle. This indicates a phase advance of the camber variation with respect to the wing pitching angle. The resulting total force $\left(F_{\text {total }}\right)$ normal to the undeformed wing surface are divided into the aerodynamic force $\left(F_{\text {aero }}\right)$ from the UAM calculation and inertial force $\left(F_{\text {inert }}\right)$ simplified as $-m \cdot a$ as shown in Figure 20(b). Since the wing camber variation is mainly due to the skin deformation under the aerodynamic pressure on the wing surface, the phase advance of wing camber variation agrees very well with the aerodynamic force.

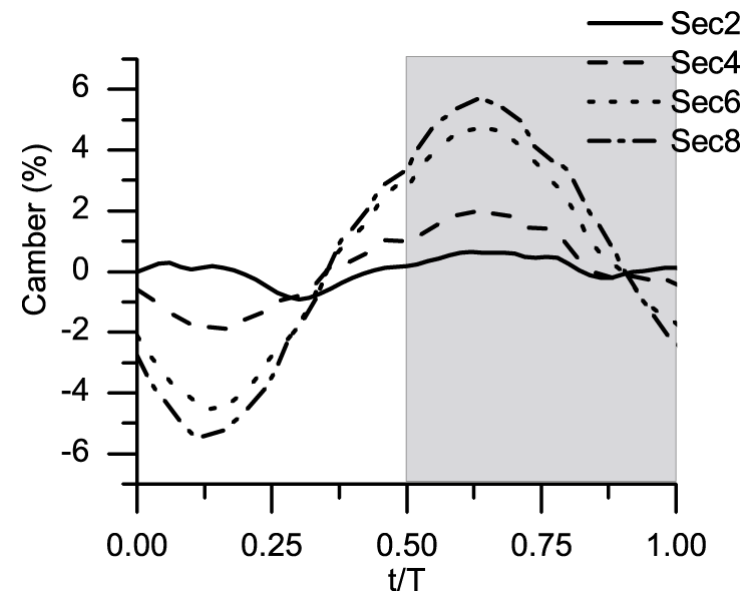

(a)

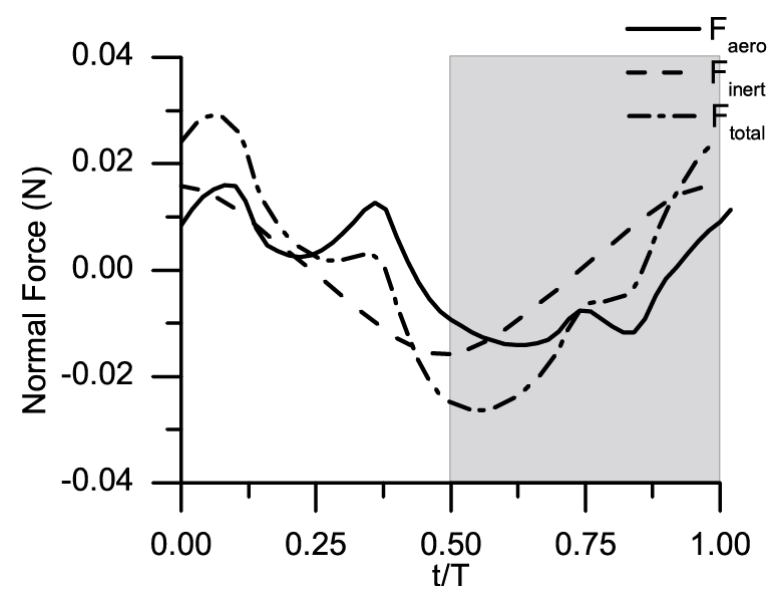

(b)

Figure 20. (a) wing camber variation of the spanwise sections (b) resulting normal forces on the wing The time courses of the twist angles of those wing sections are shown in Figure 21(a). It is noted that negative and positive twist angles mainly occur in the downstroke and upstroke respectively, and increase from the wing root to tip. This is because the flapping wing is constrained at the root of leading edge beam and cross beam, and the aerodynamic center is behind the wing elastic center. Similar to the camber variation, a phase advance for the twist angles with respect to wing pitching is observed. Figure 21(b) shows the total pitching angle of the wing at the wing tip section 8 and the elastic (deformation) twist angle separated from the prescribed sinusoidal rigid body pitching angle defined in equation (32). 


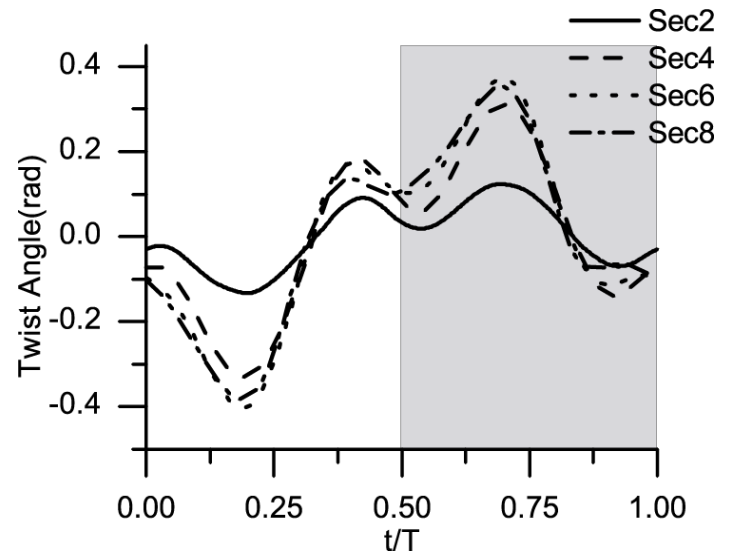

(a)

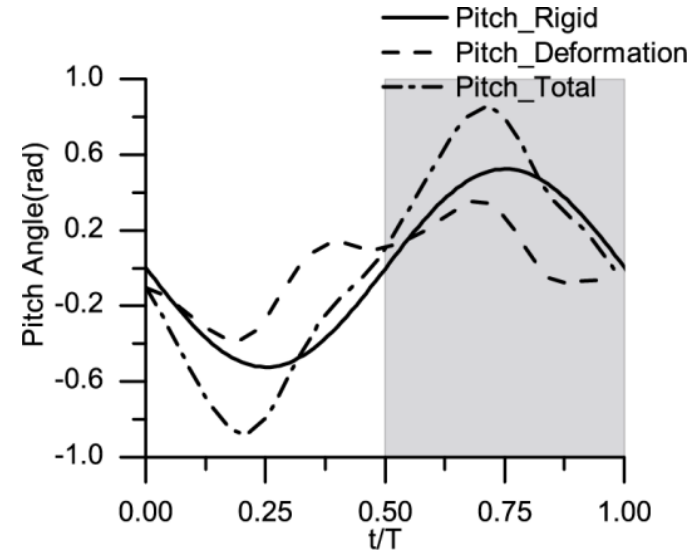

(b)

Figure 21. (a) Variation of twist angles at spanwise wing sections (b) rigid pitching angle and elastic twist angle as part of the total pitching angle

The aerodynamic lift coefficient in a flapping cycle is calculated and shown in Figure $22\left(\mathrm{C}_{L} \_\right.$Flexible $)$ based on the flapping motion and deformations of the flexible wing as shown in Figure 20 and 21. For comparison purpose, the lift coefficient of the rigid flat wing $\left(\mathrm{C}_{L_{-}}\right.$Rigid $)$as baseline case and the flexible wing in other two types of deformation models are also calculated and shown in Figure 22. The $\mathrm{C}_{L_{-}}$Camber_only is the result from the flexible wing with only the camber variation taken into account; the $C_{L} \_$Flexible_Asymmetry is from the wing of rigid flat surface in downstroke but elastic deformation including camber and twist in upstroke.

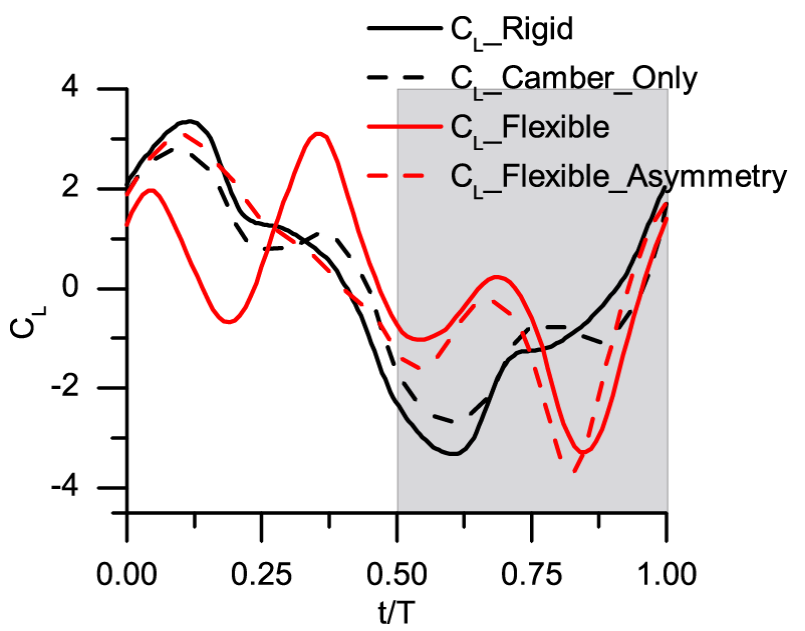

Figure 22. Comparison of time courses of lift coefficients of the wing in four different deformations

As shown in Figure 22, the $C_{L_{-}}$Camber_Only differs slightly from the $C_{L_{-}}$Rigid in both upstroke 16 and downstroke. The $C_{L \_}$Flexible has significant difference from the $C_{L \_}$Rigid in both upstroke and 17 downstroke. It is also noted that the flexible wing produces a couple of lift coefficient peaks in both 
the downstroke and upstroke compared with the typical one peak per stroke of a rigid wing. This may be caused by the phase advance of the twist angle that makes the effective AoA reduced at middownstroke and then increased before the end of downstroke.

For the above four wing models, the mean lift coefficients $\bar{C}_{L}$ in upstroke are listed in Table 5. The results show that the wing of camber variation only produces a smaller negative $\bar{C}_{L}=-1.26$ than the rigid wing $\bar{C}_{L}=-1.33$ in upstroke. The resulting $\bar{C}_{L}=-1.01$ has been further reduced when both camber and twist deformation are taken into account for the flexible wing. For the wing having asymmetric deformation, similar $\bar{C}_{L}=-1.07$ is obtained with a reduction by $19.5 \%$ comparing with the rigid wing.

The above results affect the resulting $\bar{C}_{L}$ of a complete flapping cycle of the four wing models. As shown in Table 5 , the $\bar{C}_{L}$ of the asymmetric flexible wing $\bar{C}_{L}=0.13$ is significantly larger than the other models. This is because this wing produces almost the same lift as the rigid wing in downstroke and a smaller negative $\bar{C}_{L}=-1.01$ in upstroke. The negligible small difference for the rigid and camber only wing models is due to the numerical accuracy since their $\bar{C}_{L}$ should be zero in theory. For the flexible wing, the noticeable small $\bar{C}_{L}=0.027$ may be caused by small asymmetric deformation of the wing structure with the membrane skin on one side of beams.

Table 5. Mean lift coefficient of the wing models during the upstroke only and in one flapping cycle

\begin{tabular}{|l|c|c|c|c|}
\hline & Rigid & Camber only & Flexible & $\begin{array}{c}\text { Asymmetric } \\
\text { flexible }\end{array}$ \\
\hline$\overline{\boldsymbol{C}}_{\boldsymbol{L}}$ (upstroke) & -1.33 & -1.26 & -1.01 & -1.07 \\
\hline$\overline{\boldsymbol{C}}_{\boldsymbol{L}}$ (one cycle) & 0.0141 & 0.0152 & 0.027 & 0.13 \\
\hline
\end{tabular}

\section{Conclusion}

In this study, a nonlinear unsteady aerodynamic model (UAM) based on potential flow theory for a 2D rigid airfoil section of a flapping wing has been extended. The extended UAM has improved accuracy and capability of dealing with the vortices penetration problem in an effective way, extending the analysis of a $2 \mathrm{D}$ rigid airfoil to one with variable camber, and then a flexible $3 \mathrm{D}$ wing. The extended UAM has been validated through case study of examples from CFD method and experiment available in literature. For a typical 2D rigid airfoil section and 3D wing, the difference of mean lift coefficient 
in a flapping cycle obtained from the extended UAM and CFD method is within 5\%. During the flapping motion however, there is a small phase difference between the maximum instantaneous lift coefficients of the two method (e.g. $\Delta \mathrm{t} / \mathrm{T}=0.05$ for the $3 \mathrm{D}$ wing in case 3 ). This could be due to the exclusion of air viscosity at low Re number for $2 \mathrm{D}$ airfoil and ignorance of spanwise flow for $3 \mathrm{D}$ wing.

The extended UAM can be used to evaluate the effect of camber or structural flexibility on the aerodynamic performance of a flapping wing. A rigid 2D airfoil section in a symmetric heaving and pitching motion usually produces positive lift coefficient in downstroke, which is equal in absolute value to the negative value in upstroke. An effective way of obtaining positive mean lift of a wing flapping in a vertical plan is to reduce the negative lift during upstroke. Take a wing having a pitching angle $\pm 35^{\circ}$ in a flapping cycle as example. An additional positive camber variation in upstroke ( $9 \%$ camber at mid-upstroke) results in a reduction of the negative mean lift coefficient in the upstroke by $22 \%$. The CFD results show a similar effect with the negative $\bar{C}_{L}$ reduced by $21 \%$. Consequently, the mean lift coefficient of the camber wing in a flapping cycle can be significantly increased to $\bar{C}_{L}=0.117$ from 0.0197 of the rigid wing. When the pitching angle is increased, the camber effect remains in the same level.

The same effect can be shown in a 3D wing of asymmetric flexibility due to aeroelastic effect. By using the extended UAM interfaced with a structure analysis package based on FEM, the instantaneous elastic, inertia and aerodynamic forces of a 3D flexible wing made of composite beams and film skin can be calculated and updated at each time step of the analysis in a flapping cycle. When the elastic deformation of the flexible wing is taken into account in upstroke only, the negative mean lift coefficient $\bar{C}_{L}$ in the upstroke is reduced by $24 \%$, and the $\bar{C}_{L}$ in a flapping cycle is increased from almost zero to $\bar{C}_{L}=0.13$.

The above results show that the asymmetric flexibility can significantly improve the aerodynamic performance of a flapping wing in heaving and pitching motion. This is because the positive camber and twist deformation of the wing would mitigate the usual negative lift in upstroke. This structural 
property can be achieved in practice by making use of the wing elastic deformation to gain aeroelastic beneficial effect in upstroke, but constraining the deformation in downstroke. The study provides insight for design of a practical flapping wing of high aerodynamic performance.

\section{Acknowledgments}

The authors acknowledge the financial support by China Scholarship Council (CSC NO. 201606830010); Chinese SAFEA high-end foreign expert program (GDW20163500194); fundamental research funds of Shandong University (2015JC026).

\section{References}

[1] V.W. Birnbaum, Das ebene Problemdes schlagenden Flügels, Zeitschrift Für Angew. Math. Und Mech. 4 (1924) 277-292.

[2] E. Farrell Helbling, R.J. Wood, A Review of Propulsion, Power, and Control Architectures for Insect-Scale Flapping-Wing Vehicles, Appl. Mech. Rev. 70 (2018) 10801. doi:10.1115/1.4038795.

[3] M.S. McMichael, J.M., and Francis, Micro Air Vehicles - Toward a New Dimension in Flight, Darpa, Usa. (1997).

[4] M.H. Dickinson, K. Götz, Unsteady aerodynamic performance of model wings at low reynolds numbers, J. Exp. Biol. 174 (1993) 45-64. doi:10.1242/jeb.00739.

[5] C.P. Ellington, C. van den Berg, A.P. Willmott, A.L.R. Thomas, Leading-edge vortices in insect flight, Nature. 384 (1996) 626-630. doi:10.1038/384626a0.

[6] S. Guo, D. Li, J. Wu, Theoretical and experimental study of a piezoelectric flapping wing rotor for micro aerial vehicle, Aerosp. Sci. Technol. 23 (2012) 429-438. doi:10.1016/j.ast.2011.10. 002.

[7] Z.J. Wang, J.M. Birch, M.H. Dickinson, Unsteady forces and flows in low Reynolds number hovering flight: two-dimensional computations vs robotic wing experiments., J. Exp. Biol. 207 (2004) 449-460. doi:10.1242/jeb.00739.

[8] H. Li, S. Guo, Y.L. Zhang, C. Zhou, J.H. Wu, Unsteady aerodynamic and optimal kinematic analysis of a micro flapping wing rotor, Aerosp. Sci. Technol. (2016) 1-19. doi:10.1016/j.ast. 2016.12.025.

[9] M. Sun, J. Tang, Unsteady aerodynamic force generation by a model fruit fly wing in flapping motion., J. Exp. Biol. 205 (2002) 55-70.

[10] M. Sun, J.H. Wu, Aerodynamic force generation and power requirements in forward flight in a fruit fly with modeled wing motion, J. Exp. Biol. 206 (2003) 3065-3083. doi:10.1242/jeb.00517.

[11] S. Wang, X. Zhang, G. He, T. Liu, Lift enhancement by dynamically changing wingspan in forward flapping flight, Phys. Fluids. 26 (2014) 1-56. doi:10.1063/1.4884130.

[12] J. Katz, P. Allen, Low-Speed Aerodynamicspdf, 2nd ed., Cambridge Univ. Press, Cambridge, 1991.

[13] P. Hammer, A. Altman, F. Eastep, Validation of a Discrete Vortex Method for Low Reynolds Number Unsteady Flows, AIAA J. 52 (2014) 643-649. doi:10.2514/1.J052510.

[14] Uhlman, An Integral Equation Formulation of the Equations of Motion of an incompressible fluid, (1992).

[15] H.E. Taha, M.R. Hajj, P.S. Beran, State-space representation of the unsteady aerodynamics of flapping flight, Aerosp. Sci. Technol. 34 (2014) 1-11. doi:10.1016/j.ast.2014.01.011. 
[16] Z. Yan, H.E. Taha, M.R. Hajj, Geometrically-exact unsteady model for airfoils undergoing

[17] C.-M. Gordon Lam, Nonlinear Wake Evolution of Joukowski aerofoil in severe maneuver,

[18] B. Hewett, Apprent-Mass and on-board circulation of Joukowski airfoil and cascades in severe

[19] S. a. Ansari, R. Zbikowski, K. Knowles, Non-linear unsteady aerodynamic model for insect-

[20] M.H. Dickinson, F.O. Lehmann, S.P. Sane, Wing rotation and the aerodynamic basis of insect

[21] H. Hu, A.G. Kumar, G. Abate, R. Albertani, An experimental investigation on the aerodynamic

[22] G. Du, M. Sun, Effects of unsteady deformation of flapping wing on its aerodynamic forces,

[23] Z. wu Guan, Y. liang Yu, Aerodynamic mechanism of forces generated by twisting model-

[24] Z. Guan, Y. Yu, Aerodynamics and mechanisms of elementary morphing models for flapping

[25] M. Ghommem, M.R. Hajj, P.S. Beran, I.K. Puri, Role of wing morphing in thrust generation,

[26] M. Ghommem, Global optimization of actively morphing flapping wing, J. Fluids Sructures.

[27] Z.J. Wang, Two dimensional mechanism for insect hovering, Phys. Rev. Lett. 85 (2000) 2216-

[28] D. Kim, H. Choi, Two-Dimensional Mechanism of Hovering Flight by Single Flapping Wing,

[29] Jiang Hao Wu and Mao Sun, Unsteady aerodynamic forces of a flapping wing, J. Exp. Biol.

[30] K. Karamcheti, Principles of ideal-fluid aerodynamics, (1967) pp.617-623.

[31] L.M.MILNE-THOMSON, Theoretical Aerodynamics, Book. (1966) pp.83-87.

[32] H. Taha, A. Rezaei, Unsteady Viscous Lift Frequency Response Using The Triple Deck Theory, (2018) 1-16. doi:10.2514/6.2018-0038.

[33] Y. Kamisawa, K. Isogai, Optimum Flapping Wing Motions of Dragonfly, Trans. Jpn. Soc. Aeronaut. Space Sci. 51 (2008) 114-123. doi:10.2322/tjsass.51.114.

[34] A. Gogulapati, P.P. Friedmann, E. Kheng, W. Shyy, Approximate Aeroelastic Modeling of Flapping Wings in Hover, AIAA J. 51 (2013) 567-583. doi:10.2514/1.J051801. 
2018-08-17

Unsteady aerodynamic model of flexible flapping wing

Chen, Si

Elsevier

Si Chen, Hao Li, Shijun Guo, et al., (2018) Unsteady aerodynamic model of flexible flapping wing. Aerospace Science and Technology, Volume 80, September 2018, pp. 354-367

https://doi.org/10.1016/j.ast.2018.07.017

Downloaded from Cranfield Library Services E-Repository 